\title{
Option-IMPlied Intra-Horizon VALue-AT-Risk
}

May 6, 2018

\begin{abstract}
In this paper, we theoretically and empirically study the intra-horizon value at risk (iVaR) in a general jump-diffusion setting. We propose a new class of models of asset returns, the displaced mixed-exponential model (D-MEM), which can arbitrarily closely approximate finite- and infiniteactivity Lévy processes. We then derive analytical results for the iVaR and disentangle, in a theoretically consistent way, the jump and diffusion contributions to the intra-horizon risk. We estimate historical and option-implied VaR and iVaR for several popular jump models using the S\&P 100 index and American options. Empirically disentangling the contribution of the jumps from the contribution of the diffusion, we conclude that jumps account for about 90 percent of the iVaR on average. Our backtesting results indicate that the option-implied estimates are much more responsive to market changes than their historical counterparts, which perform poorly.
\end{abstract}

Keywords: value at risk, intra-horizon risk, displaced mixed-exponential model, first-passage disentanglement, option-implied estimates.

JEL classification: G01, G11, G13, C51, C52. 


\section{Introduction}

Value at risk (VaR), defined as the conditional quantile of the profit-and-loss distribution at the end of a predefined time horizon, has been one of the most popular market risk measure for two decades now. Although widely used in practice, it does not perfectly describe the multifaceted nature of market risk. First, it is uninformative about the expected magnitude of losses beyond the calculated threshold level ${ }^{1}$ Second, the VaR captures only end-of-horizon effects. It remains silent about potential losses before the expiration of the monitored period. This aspect of risk measures has attracted much less attention 2 The goal of this paper is to study in a general jump-diffusion setting an intra-horizon value at risk (iVaR), which reflects the probability of incurring a loss of a certain size at any point in time before (and including) the end of the monitored period.

In a recent study, Bakshi and Panayotov (2010) show that, based on historical estimation, the presence of jumps tends to amplify intra horizon risk and generates large variations in risk measures across different jump models, indicating substantial model risk. Therefore, we ask whether we can improve on the historical risk estimates by using option market data and whether jump model risk, as reported in Bakshi and Panayotov (2010), still plays a dominant role. However, the analysis of jumps on option-implied intra horizon risk for different models within a unifying framework poses an additional theoretical challenge. Hence, this paper extends previous literature at least along the following dimensions.

Our first contribution is theoretical in nature. We introduce a class of exponential Lévy models, which generalizes the mixed-exponential model (MEM) studied in Cai and Kou (2011) and includes both finite and infinite activity models. We christen our new modeling framework the class of

\footnotetext{
${ }^{1}$ By now, there exists a large body of literature that addresses this issue. For example, Artzner et al. (1999), Acerbi and Tasche (2002), and Rockafellar and Uryasev (2002) developed a new risk measure, the expected shortfall (ES), which represent the average loss beyond the VaR level.

${ }^{2}$ Notable exceptions are Kritzman and Rich (2002), Boudoukh et al. (2004), Rossello (2008), Bhattacharyya et al. (2009) and Bakshi and Panayotov (2010). The intra-horizon risk was originally studied in Stulz (1996), in the context of cash flow risk in corporate risk management. Nevertheless, the notion of intra-horizon risk is much broader and has many potential applications in finance. Kritzman and Rich (2002), pp. 92-93, mention the following examples: fiduciary asset management (due to intra-horizon performance provisions), loan agreements (due to mandatory reserves covenants), hedge-fund solvency (due to possible within-horizon withdrawals), regulatory requirements (due to the maintenance of the capital account), and securities lending (due to the required collateral deposit). Therefore, the intra-horizon risk is very important in a mark-to-market environment where large trading losses in a short period of time can trigger margin calls and similar provisions.
} 
displaced mixed-exponential models (D-MEM). The D-MEM class is indeed very wide and flexible, as it can approximate processes with completely monotone Lévy densities and jump-diffusion processes with arbitrary jump distributions. Formulating different jump-diffusion models within the D-MEM class brings the advantage that we can disentangle the contribution of jumps and the diffusion term to VaR and iVaR within a unifying framework. Indeed, only with our D-MEM framework, we can shed further light on the importance of jumps for intra horizon risk across a wide range of models.

Bakshi and Panayotov (2010) established a link between the VaR (iVaR) and the expectation of a European (one-touch) digital payoff. However, since their risk calculations are based on historical data, they do not face the challenge of how to extract intra horizon information from the option market. Hence, since we cannot rely on European options for this purpose, we establish a link between the early exercise premium of American options and the first-passage time. This is our second contribution.

Bakshi and Panayotov (2010) subsequently studied VaR and iVaR risk measures for several Lévy models ${ }^{3}$ To facilitate the computations with digital payoffs, they rely on an explicit finite difference scheme. One of the main reasons for such an approach is the lack of alternative techniques that would allow studying the first-passage distributions for a wide class of exponential Lévy processes. With our D-MEM class, however, we can fill this gap. We derive analytical expressions for the expectations of European and one-touch digital payoffs in the D-MEM class using the Laplace-Carson transform (LCT). The LCT allows us to obtain a disentanglement result for the D-MEM class which enables us to consistently separate between jump and diffusion contributions to intra horizon risk across different jump models.4

Our final contribution is empirical in nature. While Bakshi and Panayotov (2010) use historical returns to analyze the potential impact of model misspecification, we take a forward-looking perspective and include information from option markets. The historical risk estimates play the role of a benchmark. Unlike most studies on the informational content of option prices, we need to use

\footnotetext{
${ }^{3}$ Other models have also been studied in the literature. Kritzman and Rich (2002) and Boudoukh et al. (2004) considered the Black-Scholes model and derived a closed-form expression for the probability of an interim loss of a given magnitude. Rossello (2008) and Bhattacharyya et al. (2009) studied the first-passage distribution in the doubleexponential jump-diffusion setting and a GARCH model with non-normal innovations, respectively, using Monte Carlo methods.

${ }^{4}$ We thereby generalize the disentanglement result obtained by Leippold and Vasiljević (2017).
} 
American options for our purpose because they are path-dependent. Market quotes of American option prices embed the information about the set of events that might occur prior to the expiry. By decomposing American option into an early exercise option and a down-and-out European put, we can link the risk-neutral probability of an early exercise to the iVaR level by applying the appropriate measure change.

For our empirical analysis, we use data on S\&P 100 index returns and American options on the S\&P 100 index. The existence of liquid options with different maturities and strikes makes it possible to study the option-implied risk-neutral probability distribution function and its moments for different investment horizons. For risk management, risk figures such as iVaR and VaR should be calculated under the physical probability measure. To transfer the calibrated risk-neutral dynamics back to the dynamics under the physical measure, we need to impose an additional assumption on the expected drift of the underlying asset. For VaR and iVaR calculations, we follow a widely accepted approach applied in previous research, which is to set the expected drift over the risk horizon (typically ten days) equal to zero. In this way, we obtain the option-implied iVaR and VaR figures under the physical probability measure. We then benchmark them against the risk estimates based on historical return data and under the same zero-drift assumption 5

To make our empirical results comparable to those of Bakshi and Panayotov (2010), we study the jump-diffusion model of Merton (1976) (the MJD), the finite-moment log-stable model (FMLS) of Carr and Wu (2003), and the Carr-Geman-Madan-Yor model (CGMY) of Carr et al. (2002). Additionally, we consider the variance gamma model (VG) of Madan and Seneta (1990). All these models can be approximated by our D-MEM class. Hence, we are able to analyze them consistently in a unified framework using our theoretical results. The model parameters are separately estimated from the historical time series of returns and the short-term American put options on the S\&P 100 index spanning the period from March 2001 until August 2014. Our empirical results for the ten-day VaR and iVaR estimates produce significantly higher values under the option-implied measure than under the historical measure.

\footnotetext{
${ }^{5}$ The zero-drift assumption for short monitoring horizons (like ten days) is used, e.g., in Boudoukh et al. (2004) and Bakshi and Panayotov (2010). We focus on the ten-day horizon, since this horizon is required by regulators for market risk calculations. As a robustness check, we also perform a backtesting analysis when we set the expected drift equal to the sample mean.
} 
Finally, we expand on the empirical analysis of Bakshi and Panayotov (2010) by providing a backtesting study for historical and option-implied VaR and iVaR figures across different models. While we do find differences of iVaR and VaR violations across different models, these differences are small compared to those between historical and option-implied VaR and iVaR figures. Our backtesting procedure shows that, irrespectively of the model used, the option-implied VaR and iVaR estimates are considerably more perceptive and responsive to asset price fluctuations, and they produce more accurate results than do the historical estimates. For this reason, we conclude that whenever options data is available, the option-implied estimates of risk measures provide additional information that should not be neglected.

The paper is structured as follows. In Section 2, we introduce the D-MEM class of exponential Lévy models and connect the VaR and iVaR to the payoffs of European and one-touch digital puts. In Section 3, we describe the data treatment and summarize the calibration and the models' performance results. Our empirical findings for the VaR and iVaR are discussed in Section 4 . We conclude in Section 5 .

\section{Theoretical Results}

To empirically study the impact of jump risk to VaR and iVaR across different models, we first introduce a unifying theoretical framework allowing us to disentangle jump and diffusion contributions for a general class of jump-diffusion models.

\subsection{Displaced mixed-exponential model (D-MEM)}

In the class of displaced mixed-exponential models (D-MEM), the asset price $S_{t}$ has the following dynamics under the physical probability measure $\mathbb{P}$ :

$$
\frac{d S_{t}}{S_{t^{-}}}=\mu d t+\sigma d W_{t}+d\left(\sum_{i=1}^{N_{t}}\left(V_{i}-1\right)\right)
$$

where $\mu \in \mathbb{R}$ and $\sigma \in \mathbb{R}^{+}$are assumed to be constant, and $\left\{W_{t}, t \geq 0\right\}$ denotes a standard Brownian motion, $\left\{N_{t}, t \geq 0\right\}$ is a Poisson process with jump intensity parameter $\lambda \in \mathbb{R}_{0}^{+}$, and $\left\{Y_{i}:=\log \left(V_{i}\right)\right.$ : 
$i=1,2, \ldots\}$ represents a sequence of independent and identically distributed (i.i.d.) random variables following a displaced mixed exponential distribution, defined below. All sources of randomness $W_{t}$, $N_{t}$, and $Y_{i}$ are assumed to be independent.

Definition 1 (Displaced mixed-exponential model). We define the class of displaced mixedexponential models (D-MEM) by specifying the Lévy density of $Y_{i}$ as

$$
\nu(y)=\lambda_{+} \sum_{i=1}^{m} p_{i} \eta_{i} e^{-\eta_{i}(y-\xi)} \mathbb{1}_{\{y \geq \xi\}}+\lambda_{-} \sum_{j=1}^{n} q_{j} \theta_{j} e^{\theta_{j}(y-\xi)} \mathbb{1}_{\{y<\xi\}} .
$$

The parameters $\lambda_{+} \in \mathbb{R}_{0}^{+}$and $\lambda_{-} \in \mathbb{R}_{0}^{+}$represent the respective (finite-activity) jump intensities of the two jump mixtures. The total jump activity is therefore $\lambda=\lambda_{+}+\lambda_{-}$. The displacement parameter $\xi$ is the mode of the Lévy density. ${ }^{6}$ The parameters $\left\{\eta_{i} \in(1,+\infty): i=1, \ldots, m\right\}$ and $\left\{\theta_{j} \in(0,+\infty): j=1, \ldots, n\right\}$ are the magnitude parameters of the jumps $\xi^{+}$and $\xi^{-}$, respectively $\rfloor^{7}$ Without loss of generality we assume that $\eta_{1}<\eta_{2}<\ldots<\eta_{m}$ and $\theta_{1}<\theta_{2}<\ldots<\theta_{n}$. The mixing weights $\left\{p_{i} \in \mathbb{R}: i=1, \ldots, m\right\}$ and $\left\{q_{j} \in \mathbb{R}: j=1, \ldots, n\right\}$ satisfy the two equations $\sum_{i=1}^{m} p_{i}=1$ and $\left.\sum_{j=1}^{n} q_{j}=1\right]^{8}$

The D-MEM in Definition 1 generalizes the mixed-exponential model (MEM) studied in Cai and Kou (2011). Indeed, with D-MEM we can approximate processes with completely monotone Lévy densities and jump-diffusion processes with arbitrary jump distributions. Therefore, several popular jump-diffusion models can be nested. Besides MEM, we can also characterize the class of hyper-exponential models (HEM) and the double-exponential models (DEMs) ${ }^{9}$

An attractive feature of the D-MEM class is that the cumulant generating function (CGF) of the

\footnotetext{
${ }^{6}$ The displacement parameter $\xi$ represents the jump magnitude at which a mixture of standard exponential distributions (the right half of the D-MEM distribution) is glued back-to-back to a mixture of " $y$-axis-mirrored" exponential distribution (the left half of the D-MEM distribution).

${ }^{7}$ The average jump size of a given type is the inverse of the corresponding magnitude parameter.

${ }^{8}$ The jump size probability distribution function (PDF) is defined as $f_{Y}(y):=\nu(y) / \lambda$. Since we allow the weights to be negative, certain conditions need to be satisfied to ensure that the function $f_{Y}(y)$ represents a PDF. Steutel (1967) showed that the following are necessary conditions $p_{1}>0, q_{1}>0, \sum_{i=1}^{m} p_{i} \eta_{i} \geq 0$, and $\sum_{j=1}^{n} q_{j} \theta_{j} \geq 0$. Bartholomew (1969) showed that the following are sufficient conditions $\sum_{i=1}^{m^{\prime}} p_{i} \eta_{i} \geq 0$ for all $m^{\prime}=1,2, \ldots, m$, and $\sum_{j=1}^{n^{\prime}} q_{j} \theta_{j} \geq 0$, for all $n^{\prime}=1,2, \ldots, n$.

${ }^{9}$ The HEM class of models was studied in Cai (2009, 2011), Crosby et al. (2010), Jeannin and Pistorius (2010), Boyarcheko and Boyarchenko (2011), Cai and Kou (2012), among others. The main references for DEMs are Kou|(2002), Kou and Wang (2003, 2004), Sepp (2004), AitSahlia and Runnemo (2007), Ramezani and Zeng (2007), Bayraktar and Xing (2009, 2011), Albrecher et al. (2012), among others.
} 
$\log$-price process $\left\{X_{t}:=\log S_{t}, t \geq 0\right\}$ is given in analytical form, i.e.,

$$
\Psi(u):=\frac{1}{t} \log \mathbb{E}\left[e^{u X_{t}}\right]=\bar{\mu} u+\frac{1}{2} \sigma^{2} u^{2}+\lambda \zeta(u), \quad u \in\left(-\theta_{1}, \eta_{1}\right),
$$

where $\bar{\mu}:=\mu-\lambda \zeta(1)-\frac{\sigma^{2}}{2}$ represents the compensated drift term and $\zeta(1)$ is the average jump size. The function $\zeta(u)$ is given by

$$
\zeta(u):=\mathbb{E}\left[e^{u Y_{1}}-1\right]=\left(\frac{\lambda_{+}}{\lambda} \sum_{i=1}^{m} \frac{p_{i} \eta_{i}}{\eta_{i}-u}+\frac{\lambda_{-}}{\lambda} \sum_{j=1}^{n} \frac{q_{j} \theta_{j}}{\theta_{j}+u}\right) e^{u \xi}-1 .
$$

The D-MEM class serves as a unifying framework to study different jump diffusion models. In particular, as we show below, it allows us to disentangle the jump from the diffusion component of the iVaR within the same model class 10 For our empirical study, we consider as candidate jump diffusion models the Merton jump-diffusion (MJD), the finite-moment log-stable (FMLS), the variance gamma (VG), and the Carr-Geman-Madan-Yor (CGMY) model. With D-MEM we can arbitrarily closely approximate all these models. We detail these approximations in Appendix B.

For our endeavor, we not only need the physical probability measure to calculate VaR and iVaR risk figures, but we also need the pricing measure to calibrate our model to option data. Therefore, we need to specify the corresponding measure change. Given the inherent market incompleteness of Lévy models, we make use of the transform of Esscher (1932), which admits a structure-preserving change of measure for D-MEM processes. Details are given in Proposition A.1 of Appendix A.

We recall that in Equations (1) and (2), we formulated the D-MEM class under the physical measure $\mathbb{P}$. For risk management, it is obvious that we want to calculate iVaR and VaR figures under the measure $\mathbb{P}$. However, the true data-generating process under $\mathbb{P}$ is not observable. Therefore, we focus on two alternative strategies to estimate the underlying dynamics. In the first one, we follow Bakshi and Panayotov (2010) and use historical data to nail down the parameters of the model. In the second one, we calibrate our models to options data under the measure $\mathbb{Q}$. With the results in

\footnotetext{
${ }^{10}$ One could argue we could estimate VaR and iVaR using a different approach, e.g., by numerically solving the partial integro-differential equation as in Bakshi and Panayotov (2010) or by using Fourier methods. Therefore, we could estimate our risk measures without invoking the D-MEM model. However, if we would do so, we could no longer disentangle jumps from diffusion in a theoretically consistent way. While our disentanglement results in the D-MEM framework are exact and intuitive, any method relying on a discretization of the underlying price space cannot provide a reliable decomposition of diffusion and jump contributions to the iVaR.
} 
Proposition A.1, we can then transform back the $\mathbb{Q}$-dynamics to the option-implied $\mathbb{P}$-dynamics.11 However, this transformation requires an assumption on the parameter $\mu$. As argued before, we adopt the assumption of Boudoukh et al. (2004) and Bakshi and Panayotov (2010) that the drift of the stock price over the VaR horizon is equal to zero, i.e., $\mu=0{ }^{12}$ Accordingly, we refer to the iVaR and VaR figures obtained by using the option-implied $\mathbb{P}$-dynamics as option-implied iVaR and VaR, contrasting the historical iVaR and VaR figures that depend on historical data. By setting $\mu=0$, both the historical and the option-implied risk figures become comparable, helping us to identify the impact of using option data.

\subsection{Linking European and American options to VaR and iVaR}

Before we can use our modeling framework to calculate option-implied iVaR and VaR from market data, we first need to establish a theoretical link between these risk measures and option prices. The payoff of an American put option depends on the first hitting time $\tau_{B}$ of the early exercise boundary $\left(B_{t}, 0 \leq t \leq T\right)$. If exercised before maturity, the payoff is equal to $K-S_{\tau_{B}}$. Otherwise, it matches the payoff of the corresponding down-and-out European put option. Therefore, an American put option with maturity $T$ and strike price $K$ can be decomposed into

$$
\begin{aligned}
\operatorname{Put}\left(S_{t} ; B\right) & =\underset{\tau_{B} \in \mathcal{T}_{[t, T]}}{\operatorname{ess} \mathbb{E}_{t}^{*}\left[e^{-r\left(\tau_{B}-t\right)}\left(K-S_{\tau_{B}}\right)^{+}\right]} \\
& =\underbrace{\mathbb{E}_{t}^{*}\left[e^{-r\left(\tau_{B}-t\right)}\left(K-S_{\tau_{B}}\right) \mathbb{1}_{\left\{\tau_{B}<T\right\}}\right]}_{\text {Early exercise option (EEO) }}+\underbrace{\mathbb{E}_{t}^{*}\left[e^{-r(T-t)}\left(K-S_{T}\right)^{+} \mathbb{1}_{\left\{\tau_{B} \geq T\right\}}\right]}_{\text {Down-and-out European put }},
\end{aligned}
$$

where $\mathbb{E}_{t}^{*}$ denotes the expectation under $\mathbb{Q}^{\vartheta}$ conditional on $\mathcal{F}_{t}$. With the barrier decomposition in Equation (5), we can isolate the intra-horizon effect through the early exercise option (EEO) 13

Following the constant barrier approach of Broadie and Detemple (1996) and Ingersoll (1996), we approximate the true early exercise boundary $B$ with a flat early exercise policy $L$. With such an approximation, we obtain an admissible theoretical price of an uncapped American option by

\footnotetext{
${ }^{11}$ The option-implied iVaR and VaR should therefore not be confused with the risk-neutral risk figures, as we transform the dynamics back from $\mathbb{Q}$ to $\mathbb{P}$.

${ }^{12}$ Hence, the Esscher transform parameter $\vartheta$ is uniquely determined, see Appendix A Equation A.4 for details.

${ }^{13}$ An alternative decomposition of American options is based on the early exercise premium. However, the early exercise premium comprises two components, one of which depends on an end-of-horizon event, which hinders analysis of "pure" intra-horizon risk.
} 
maximizing a capped option price over the level $L$. Therefore, denoting the price of a capped American put option by $\overline{\mathrm{Put}}\left(S_{t} ; L\right)$, we obtain the optimal value of the level parameter as

$$
L^{\star}=\underset{L \leq S_{t}}{\arg \max } \overline{\operatorname{Put}}\left(S_{t} ; L\right), \quad \text { s.t. } 0<L \leq K .
$$

Using $\overline{\mathrm{Put}}\left(S_{t} ; L^{\star}\right)$ as an approximation for the uncapped American put, the early exercise option can be further decomposed as

$$
\begin{aligned}
\operatorname{EEO}\left(S_{t} ; L^{\star}\right) & :=\mathbb{E}_{t}^{*}\left[e^{-r\left(\tau_{L^{\star}}-t\right)}\left(K-S_{\tau_{L^{\star}}}\right) \mathbb{1}_{\left\{\tau_{\left.L^{\star}<T\right\}}\right]}\right] \\
& =K \underbrace{\mathbb{E}_{t}^{*}\left[e^{-r\left(\tau_{L^{\star}}-t\right)} \mathbb{1}_{\left\{\tau_{\left.L^{\star}<T\right\}}\right.}\right]}_{\text {One-touch digital option }}-\mathbb{E}_{t}^{*}\left[e^{-r\left(\tau_{L^{\star}}-t\right)} S_{\tau_{L^{\star}}} \mathbb{1}_{\left\{\tau_{L^{\star}}<T\right\}}\right] .
\end{aligned}
$$

The first term in Equation (7) represents a one-touch digital option, which provides us with a direct link to the probability of an early exercise.

We recall from Bakshi and Panayotov (2010) that iVaR, defined as the conditional quantile of the first-passage distribution (FPD) of the asset price process $\left\{S_{u}, t \leq u \leq T\right\}$, is related to the cumulative distribution function $(\mathrm{CDF})$ of the FPD evaluated at a certain pre-specified level $K$ :

$$
P\left(S_{t}, \tau ; K\right):=\mathbb{E}_{t}\left[\mathbb{1}_{\left\{S_{u}<K\right\}}, t<u \leq T\right]
$$

where $\tau:=T-t$ represents the remaining time before the expiry of the monitoring period, and the asset price at time $t$ is given by $S_{t}$. In particular, the iVaR with the monitoring horizon $\tau$ and the confidence level $\chi$ can be computed as

$$
\operatorname{iVaR}(\tau, \chi)=S_{t}-\underline{K}, \text { given that } P\left(S_{t}, \tau ; \underline{K}\right)=1-\chi
$$

i.e., the iVaR corresponds to the difference between the current asset price $S_{t}$ and the implied $(1-\chi)$ quantile level $\underline{K}$. With this insight, and neglecting the discount factor for the usual 10-day horizon used in VaR calculations, we apply the measure change in Proposition A.1 to transform the one-touch digital option under $\mathbb{Q}^{\vartheta}$ in Equation (7) into the CDF of the FPD under $\mathbb{P}$ in Equation (8). Such a procedure allows us to use data from option markets to derive option-implied iVaR figures.

Similarly, the VaR is related to the CDF of the asset price process at the end of the monitoring 
period $\tau$, which can be computed as an expectation of the European digital put payoff:

$$
p\left(S_{t}, \tau ; K\right)=\mathbb{E}_{t}\left[\mathbb{1}_{\left\{S_{T}<K\right\}}\right],
$$

where $K$ is the strike price. Therefore, the VaR can be computed as the difference between the current asset price $S_{t}$ and the implied $(1-\chi)$-quantile level $\bar{K}$ of the asset price CDF at the end of the monitoring period:

$$
\operatorname{VaR}(\tau, \chi)=S_{t}-\bar{K}, \text { given that } p\left(S_{t}, \tau ; \bar{K}\right)=1-\chi
$$

Equations (9) and (11) can be solved using, e.g., the bisection method. It can be verified that

$$
\operatorname{VaR}(\tau, \chi)<\operatorname{iVaR}(\tau, \chi) \text {, a.s. for } \tau \in \mathbb{R}^{+}, \chi \in(0,1) \text {. }
$$

To calculate the option prices that provide a link to VaR and iVaR, we use the Laplace-Carson transform (LCT) approach. The main idea of our solution procedure is to compute the LCT of the option price with respect to the monitoring horizon $\tau$. The Laplace-Carson transform has the interpretation of a so-called Canadized option, e.g., see Carr (1998). As we show in Appendix A.2, we can calculate Canadized options in analytical form, which then allows us to use the Gaver-Stehfest inversion algorithm to efficiently calculate the prices of American put options with finite maturity. The corresponding formulas are given in Appendix A.2.

Lastly, to isolate the effect of the jump components on VaR and iVaR figures across different model specifications within the D-MEM class, we need to generalize the disentanglement results of Leippold and Vasiljević (2017) to the D-MEM class. To this end, we recall that a one-touch digital put is exercised if the asset price directly hits or overshoots the barrier at any point in time before maturity. Due to its almost sure continuity of paths, and conditionally on the stopping of the process, a Brownian motion almost surely hits the barrier separating the continuation from the stopping region. On the other hand, an overshoot occurs if the asset price jumps over the barrier directly into the interior of the stopping region. Conditional on the stopping of the process due to a

\footnotetext{
${ }^{14}$ Intuitively, the probability of crossing a barrier level (from above) at any point in time during the monitoring period is higher than the probability that the underlying process will end up below the barrier level at the expiration date. Indeed the process which is in the stopping region at the end of the monitoring period has almost surely breached the barrier level before the expiration. Therefore, if a European and a one-touch digital put have identical prices, i.e., $p\left(S_{t}, \tau ; \bar{K}\right)=P\left(S_{t}, \tau ; \underline{K}\right):=1-\chi$, then almost surely the implied barrier level $\underline{K}$ (the iVaR) has to be lower (greater) than the implied strike $\bar{K}$ (the VaR).
} 
jump event, continuously distributed jumps almost surely induce an overshoot, i.e., the price process will almost surely not jump onto the barrier. We use this insight to derive the disentanglement results in Proposition A.4, which finally allows us to quantify the first-passage disentanglement of the jump from the diffusion contribution to the stopping of the process, hence to the price of a one-touch digital put and the iVaR value.

We note that the impact of jumps on American option prices has been recently considered in Chiarella et al. (2009). They examine jump effects by comparing the shape of the early exercise boundary with and without jumps, keeping the overall volatility constant. In contrast, Proposition A.4 allows us to disentangle jumps from the diffusion component within the same model, without having to impose some moment conditions. Hence, our procedure provides a consistent comparison of jump and diffusion contributions to iVaR across different models within the D-MEM class.

\section{Calibrating jump models}

To calibrate the candidate jump models, the Merton jump-diffusion (MJD), the finite-moment logstable (FMLS), the variance gamma (VG), and the Carr-Geman-Madan-Yor (CGMY) model, under both the physical and risk-neutral measures, we use two different data sets: the historical returns of the S\&P 100 index and OEX American options quotes ${ }^{15}$ All the data were obtained from OptionMetrics.

\subsection{Data}

To calculate the historical iVaRs, we use a time series of 939 weekly historical returns of the S\&P 100 index from January 1996 until August 2014. Hence, our data spans almost two decades and encompasses a broad spectrum of market conditions, including the recent global financial crisis. The data treatment for the historical returns time series closely follows Bakshi and Panayotov (2010). We re-estimate the models on a monthly basis, and create moving/rolling windows of 260 weekly

\footnotetext{
${ }^{15}$ Similarly to Bakshi and Panayotov $\sqrt{2010)}$, the dividends are neglected in our study.
} 
returns. This implies that the first month for which we estimate the models will be March 2001 because we need five years of data to create a weekly time series of desired length.

To calculate the option-implied iVaR, we use S\&P 100 index American options data (ticker symbol: OEX), spanning the period from March 2001 until August 2014. The starting month of our options sample coincides with the first month for which we are able to estimate the models based on the time series of S\&P 100 historical returns, conditional on the monthly re-calibration procedure and the choice of weekly sampling frequency. Overall, this leaves us with 162 dates on which we estimate the models on both the historical and the options data. For re-calibration timestamps, we choose those dates at the beginning of each month on which there exist liquid options maturing in exactly ten trading days, hence matching the VaR/iVaR horizon.

For each date included the sample, the options data treatment is conducted as follows. We define the moneyness $m$ as the ratio of the strike $K$ and the futures price $F_{t, T}:=S_{t} e^{r(T-t)}$, i.e., $m:=K / F_{t, T}$. In the first step, we eliminate all calls because by construction puts are much more informative of the downside risk in the underlying ${ }^{16}$ Second, we exclude illiquid in-the-money put options (ITM), i.e., puts with moneyness greater than 1.03. Hence, our sample contains only liquid near-the-money (NTM) and out-of-the-money (OTM) put options. To match the ten-day horizon for the computation of the risk measures, we keep only options with maturities equal to or shorter than ten days. We use mid-prices, i.e., averages of the bid and ask market quotes, as the proxy for our market prices. Option quotes lower than 0.125 units are eliminated due to the minimum tick limitations. Additionally, we eliminate the options with zero volume or open interest.

[Table 1 about here.]

Descriptive statistics of our option sample are given in Table1. The total number of option quotes in the dataset (spanning 162 days) is 3,411. NTM put options, i.e., options for which $m \in(0.97,1.03)$, account for approximately 41.4 percent of the sample. OTM puts $(m<0.97)$ constitute around 58.6 percent of the dataset. The average implied volatility smile ranges from 21.15 percent (NTM puts) to 39.78 percent (OTM puts).

\footnotetext{
${ }^{16}$ For simplicity, we consider only the long position in the S\&P 100 index, and therefore focus on the left tail of the distribution.
} 


\subsection{Calibration results}

First, we estimate the parameters for each of the considered models under the historical measure, which will serve as the benchmark in our empirical study. In particular, we conduct a rolling-window maximum likelihood estimation (MLE) using weekly historical returns. However, there are notable differences in our estimation approach from that of Bakshi and Panayotov (2010). First, we use the Fourier cosine method of Fang and Oosterlee (2008) to compute the probability distribution function (PDF) of the log-price process. Second, they restrict the value of the fine structure parameter $Y$ to 0.5 in the CGMY model. In contrast, we allow for all values in the interval $Y \in(-1,2)$, which ensures an additional degree of freedom in the CGMY model. Finally and most importantly, we also use data from options markets. The calibration under the risk-neutral measure is conducted by minimizing a loss function in the form of a weighted non-linear least squares (WNLLS) for each date in our sample. Our objective function can be interpreted as a weighted Euclidean distance between the market quotes and the corresponding model option prices, and the weights are defined as the inverses of the squares of the bid-ask spreads.

[Table 2 about here.]

In Table 2, we report the average values and standard deviations of model parameters over the whole sample (162 days) for both historical and option-based calibrations. Since the mean might be influenced by outliers and non-normality, we also report the median and the median absolute deviation. 17 Several patterns can be observed across the estimates. First, the option-implied estimates exhibit larger variations across time than the parameters estimated from the historical returns estimation. Arguably, this is due to the better responsiveness of the option-implied model parameters to changing market conditions.

Second, for the infinite activity Lévy processes (FMLS, VG, and CGMY), the jump arrival rate $C$ is typically inversely proportional to the fine structure parameter $Y$. This is plausible because higher values of the fine structure parameter indicate increased activity of the small jumps, which

\footnotetext{
${ }^{17}$ The estimate for $\mu_{J}$ indeed seems to be influenced by outliers, when we compare the mean of $\mu_{J}$ under the riskneutral and the physical measure. However, for the median, which is robust to outliers, the difference between the $\mu_{J}$ 's becomes less dramatic, $-3 \%$ under the historical measure and $-5 \%$ under the risk-neutral measure.
} 
are effectively "taking over" some part of the variation of the stochastic process that would otherwise be captured by the jump intensity parameter. Third, the exponential decay parameters $M$ and $G$ are positively correlated with the parameter $C$. The reason is that the reciprocal values of $M$ and $G$ represent the average sizes of large jumps, which are inversely related to the jump intensity parameter due to the complete monotonicity. Fourth, we observe across all models that the average jump size is negative, which indicates negative skewness.

\subsection{Model performance}

Comparing the MLE figures in Table2, we find that under the historical measure the tested models exhibit similar performance. However, when we consider the models under the risk-neutral measure, a pattern emerges. Based on the MLE, the CGMY model seems to provide the best fit. For a consistent comparison of the performance of different models, we conduct Vuong (1989)'s closeness test for non-nested models and report the results in Table 3 .

[Table 3 about here.]

Panel A of Table 3 summarizes the Vuong test statistics for the historical estimation. The calibration performance ordering among the models is: VG $\succ$ FMLS $\succ$ MJD $\succ$ CGMY. On the other hand, the hierarchy of the models looks different for the option-based estimation in Panel $\mathrm{B}: \mathrm{CGMY} \succ \mathrm{MJD} \succ \mathrm{VG} \succ \mathrm{FMLS}{ }^{18}$ We therefore conclude that the model performance rankings diverge under the historical and the risk-neutral measures. This finding reinforces the importance of our research question regarding the relevance and the reliability of option-based estimates of risk measures and more generally about the impact of the estimation risk in the market risk management context.

\footnotetext{
${ }^{18}$ For a given two models $\mathrm{A}$ and $\mathrm{B}$ we write $\mathrm{A} \succ \mathrm{B}(\mathrm{B} \succ \mathrm{A})$ if the Vuong $(1989)$ 's closeness test is rejected in favor of model A (B).
} 


\section{Empirical results}

\subsection{VaR and iVaR estimates}

To estimate VaR and iVaR we proceed as follows. First, we employ the approximation procedure outlined in Appendix B to obtain the historical and the risk-neutral D-MEM parameters corresponding to the calibrated Lévy models. Second, we translate the risk-neutral parameters into their counterparts under the physical probability measure using the D-MEM change of measure in Proposition A.1 under the assumption $\mu=0$. Again, we could think of other choices of $\mu$, but we follow here the argumentation of Bakshi and Panayotov (2010).

Using the D-MEM approximation results, the iVaR and VaR estimates are computed - assuming 99.0\% and $99.9 \%$ confidence levels and a ten-day monitoring horizon-applying the pricing formulas in Propositions A.2 and A.3 respectively. Finally, for each observation date in the sample, we decompose the estimated iVaR into a diffusion and a jump component by applying the theoretical results for the first-passage disentanglement in Proposition A.4.

[Figure 1 about here.]

In Figure 1, we plot the time evolution of the jump contribution to the iVaR based on our theoretical disentanglement result in Proposition A.4. Irrespectively of the model and the estimation procedure, jumps typically account on average for around $90 \%$ of the iVaR. Hence, if the critical intrahorizon reserve capital level is breached at some point in time before the expiry of the monitoring period, it is most likely caused by a jump in the asset price process. Moreover, such a jump almost surely overshoots, i.e., the loss surpasses the predetermined set-aside cash buffer.

Following Bakshi and Panayotov (2010), we can additionally quantify the impact of the jumps and intra-horizon risk by computing the ratios of our jump models' VaR and iVaR estimates to two benchmark VaR values. ${ }^{19}$ The first benchmark is the $(1-\chi)$-quantile of the normal distribution $\mathcal{N}\left(\hat{\mu}, \hat{\sigma}^{2}\right)$, where $\chi$ is either $99.0 \%$ or $99.9 \%$. The parameter $\hat{\mu}=0$ is the drift, and the parameter $\hat{\sigma}$

\footnotetext{
${ }^{10}$ Bakshi and Panayotov $(2010)$ used the term 'multiple' for these ratios. We adopt their notation, however it should be noted that Boudoukh et al. (2004) and Rossello (2008) used the term 'inflation factor' instead.
} 
is the standard deviation of the de-meaned returns process under the historical measure. The second benchmark is constructed using the filtered historical simulation (FHS) approach of Barone-Adesi et al. (1999) and Barone-Adesi et al. (2008) ${ }^{20}$

We summarize our findings in Table 4. Panel A reports the average, median, and maximum values of multiples of the $\mathrm{VaR}$ and the $\mathrm{iVaR}$, based on the estimation under the historical measure. Compared to the findings of Bakshi and Panayotov (2010), we estimate similar or lower (similar or higher) VaR and iVaR multiples at the 99.0\% (99.9\%) confidence level. Almost uniformly, the CGMY (FMLS) model has the lowest (highest) VaR and iVaR multiples. This result is even stronger for the more stringent confidence level, i.e., the relative difference of the CGMY (FMLS) multiples with respect to the other two jump models is amplified. Elevated FMLS multiples can be explained by the fact that it is the only spectrally negative process, and the only one without exponentially damped Lévy density, among the four considered jump models. Overall, at a 99.0\% (99.9\%) confidence level, a historical VaR estimator and a historical iVaR estimator can increase the benchmark level by a factor of 1.99 (3.43) and 2.16 (3.52), respectively. Using the alternative benchmark VaR, we obtain the same rankings of the models. However, as expected, the multiples are partially damped. The maximum multiples are $1.24(2.11)$ and $1.35(2.17)$ at the $99.0 \%(99.9 \%)$ confidence level for the respective risk measures.

[Table 4 about here.]

In Panel B of Table 4, we report the same descriptive statistics based on the option-implied multiples. We immediately observe that all the statistics of the multiples are uniformly and significantly higher in this case. Therefore, they are more conservative and thus may provide better protection against adverse market moves. Moreover, the forward-looking multiples exhibit larger variation across models, which can be interpreted as the model risk. However, the stupendous difference between the multiples estimated under the two probability measures (especially for the maximum iVaR

\footnotetext{
${ }^{20}$ Our FHS exercise is based on an $\mathrm{AR}(1)-\mathrm{GARCH}(1,1)$ model with leverage effect under the historical measure; see Monfort and Pegoraro (2012). The algorithm is explained in detail in Barone-Adesi et al. (1999). We note that the GARCH model is estimated using five years of daily returns, i.e., 1,300 observations, and the FHS distributions of the cumulative returns and the running minima over a ten-day horizon (from which we compute the VaR and the iVaR, respectively) arise from 10,000 replications of the filtered historical returns simulation.
} 
multiples at the $99.9 \%$ confidence level) strongly indicates that the estimation risk is probably even more important. In terms of the option-implied rankings, the MJD (CGMY) model has the lowest (highest) VaR and iVaR multiples. Using the option-implied VaR and iVaR estimators can increase the benchmark level by a factor of 7.61 (9.96) and 8.12 (9.96), respectively, at the $99.0 \%(99.9 \%)$ confidence level. We note that the alternative VaR benchmark model yields again the same rankings. The maximum multiples with respect to the FHS VaR are 4.55 (5.64) and 4.86 (5.63) at the 99.0\% (99.9\%) confidence level under the historical and the option-implied measures, respectively.

[Figure 2 about here.]

To provide some further intuition about the behavior of multiples, we plot in Figure 2 the dynamics of the ratio of option-implied and historical iVaR estimates for each of the jump models separately ${ }^{21}$ The option-implied iVaR estimates are lower than their historical counterparts in the periods of market calm, i.e., the ratio is lower than one. Conversely, the ratio sharply rises during the financial and economic crises, most notably during the stock market downturn of 2002, the financial crisis of 2008, the European debt crisis of 2010, and the stock market downturn of 2011. Hence, the option-implied iVaR is more sensitive and responsive to market conditions, and can be interpreted as a risk measure implied by future market beliefs. In contrast, historical estimates do not properly capture these forward-looking beliefs when a crisis suddenly occurs.

\subsection{Backtesting}

For our backtesting analysis, we first provide some intuition by plotting in Figure 3 the VaR estimates under the historical and the option-implied measures with the realized ten-day cumulative returns. Additionally, we include the times series of FHS VaR estimates. An analogous plot for the iVaR is presented in Figure 4, where we use the minimum interim ten-day cumulative returns. In both cases, we only consider the $99.0 \%$ confidence level.

[Figure 3 about here.]

\footnotetext{
${ }^{21}$ The ratio of the option-implied and historical VaR estimates follows an almost identical pattern, hence we omit it in Figure 2,
} 
[Figure 4 about here.]

From Figures 3 and 4, we observe that the historical VaR and iVaR seem to underestimate the true risk, and this underestimation is exacerbated for intra horizon risk 22 In contrast, the optionimplied VaR and iVaR tend to slightly overestimate risk, at least for our sample period ${ }^{23}$ Moreover, the number of $\mathrm{VaR}$ and $\mathrm{iVaR}$ violations is the same under the option-implied measure, whereas the number of iVaR breaches is larger than the number of VaR breaches under the historical measure. This preliminary analysis may indicate that the historical approach is less effective at capturing the intra horizon component of iVaR.

To properly evaluate the model performance we consider a battery of standard statistical tests. In particular, we employ the backtesting procedures of Kupiec (1995), i.e., the Time Until First Failure test (TUFF) and the Proportion of Failures test (POF), which is also known as the Unconditional Coverage test (UC). Moreover, we include the Independence Coverage test (IC) and the Conditional Coverage test (CC) of Christoffersen (1998).

[Table 5 about here.]

The results are summarized in Table 5, and they reinforce our earlier conclusions. Irrespectively of the jump model, historical VaR models provide relatively poor risk forecasts, hence they are largely rejected by the standard statistical tests (Panel A). The performance additionally worsens in the case of iVaR backtesting (Panel C). In contrast, the option-implied approach to the estimation of the VaR and iVaR yields much better results (Panel B and Panel D, respectively). None of the models estimated under the forward-looking measure are rejected at the conventional confidence levels of $95 \%$ and $99 \%$. Nevertheless, we observe a somewhat better performance of the MJD and CGMY models in comparison to the VG and FMLS models. Hence, our results indicate that we can improve the measurement of market risk by using forward-looking information. Using historical data provides a poor risk forecast. At the same time, once we use the option-implied approach, model risk becomes less important, at least for our candidate models, which all exhibit a jump component.

\footnotetext{
${ }^{22}$ Under the historical measure, there are typically 6-7 VaR breaches and 8-11 iVaR breaches in the total of 162 observations. These numbers correspond to $96.30 \%-95.68 \%$ VaR and $95.06 \%-93.21 \%$ iVaR levels, respectively.

${ }^{23}$ There are either no violations at all or at most one VaR and iVaR violations, corresponding to $100 \%-99.38 \%$ levels and close to the target confidence level of $99 \%$.
} 
As a robustness check, we also calculated the option-implied risk estimates under different assumptions about the mean drift $\mu$. We recall that the calculations in Table 5 are based on the assumption of $\mu=0$. Another sensible choice could be the sample mean, which in our case corresponds to $\hat{\mu}=3.6 \%$ over the period March 2001-August 2014. When we recalculate the option-implied VaR and iVaR and perform the same backtesting analysis, we get additional violations for each model, but still very close to the $99 \%$ target level. Therefore, even if we choose a less conservative assumption for the mean drift by setting it equal to its historical mean, we get highly accurate results for the option-implied risk measures. In contrast, since $\mu=0$ is a conservative choice, the historical VaR and iVaR estimates under $\mu=\hat{\mu}$ will be pushed even further off the $99 \%$ target level. ${ }^{24}$

\section{Conclusion}

In this paper, we have developed a theoretical framework, the D-MEM class, which encompasses both finite- and infinite-activity jump models, for the computation of the VaR and iVaR. Additionally, we derived a new result for the disentanglement of the contribution to the iVaR of the jumps from the contribution of the diffusion. With these theoretical results, we are equipped to consistently compare the jump contributions to intra-horizon risk across different models within the D-MEM class and with information extracted from option markets.

In an empirical study, we applied our theoretical results to several popular Lévy models using historical return time series and short-term American put options on the S\&P 100 index from March 2001 to August 2014. Our theoretical results allow us to empirically disentangle the contribution to the iVaR of the jumps from that of the diffusion. We find that jumps are the main driver of the intra-horizon risk, accounting for about $90 \%$ on average. Furthermore, we observe that in times of crisis, the option-implied VaR and iVaR estimates are significantly higher than their historically estimated counterparts. At the same time, during periods of market calm, the option-implied risk estimates are lower than their historical counterparts.

The statistical backtesting procedures provide further support for the judgement that optionbased VaR and iVaR forecasts exhibit a superior forecasting performance. Moreover, the intra-

\footnotetext{
${ }^{24}$ Details for these results are not reported here, but can be obtained from the authors.
} 
horizon feature exacerbates the problems inherent to the historical approach. Our conclusion that the option-implied risk estimates provide better protection against future adverse market moves can be explained by their forward-looking nature, which ultimately renders them remarkably perceptive and responsive to financial market conditions. Overall, our empirical findings are much more sensitive to the choice of probability measure than the choice of the jump model. Therefore, within the scope of our study, the estimation risk surpasses the model risk in importance. For this reason, we conclude that the option-implied estimation of risk measures should not be neglected in practice whenever options data is available. 


\section{References}

Abate, J. and Whitt, W.: 2006, A unified framework for numerically inverting Laplace transforms, INFORMS Journal on Computing 18, 408-421.

Acerbi, C. and Tasche, D.: 2002, On the coherence of expected shortfall, Journal of Banking and Finance 26, 1487-1503.

AitSahlia, F. and Runnemo, A.: 2007, A canonical optimal stopping problem for american options under a double exponential jump-diffusion model, Journal of Risk 10, 85-100.

Albrecher, H., Kortschak, D. and Zhou, X.: 2012, Pricing of Parisian options for a jump-diffusion model with two-sided jumps, Applied Mathematical Finance 19, 97-129.

Artzner, P., Delbaen, F., Eber, J. M. and Heath, D.: 1999, Coherent measures of risk, Mathematical Finance 9, 203-228.

Asmussen, S., Madan, D. and Pistorius, M.: 2007, Pricing equity default swaps under an approximation to the CGMY Lévy model, Journal of Computational Finance 11, 79-93.

Bakshi, G. and Panayotov, G.: 2010, First-passage probability, jump models, and intra-horizon risk, Journal of Financial Economics 95, 20-40.

Barone-Adesi, G., Engle, R. F. and Mancini, L.: 2008, A GARCH option pricing model with filtered historical simulation, Review of Financial Studies 21, 1223-1258.

Barone-Adesi, G., Giannopoulos, K. and Vosper, L.: 1999, VaR without correlations for portfolios of derivative securities, Journal of Futures Markets 19, 583-602.

Bartholomew, D. J.: 1969, Sufficient conditions for a mixture of exponentials to be a probability density function, The Annals of Mathematical Statistics 40, 2183-2188.

Bayraktar, E. and Xing, H.: 2009, Pricing american options for jump diffusions by iterating optimal stopping problems for diffusions, Mathematical Methods of Operations Research 70, 505-525.

Bayraktar, E. and Xing, H.: 2011, Pricing Asian options for jump diffusion, 21, 117-143. 
Bhattacharyya, M., Misra, N. and Kodase, B.: 2009, Maxvar for non-normal and heteroskedastic returns, Quantitative Finance 9, 925-935.

Botta, R. F. and Harris, C. M.: 1986, Approximation with generalized hyperexponential distributions: weak convergence results, Queueing Systems 1, 169-190.

Boudoukh, J., Richardson, M., Stanton, R. and Whitelaw, R. F.: 2004, MaxVaR: Long-horizon value at risk in a mark-to-market environment, Journal of Investment Management 2, 1-6.

Boyarcheko, M. and Boyarchenko, S.: 2011, Double barrier options in regime-switching hyperexponential jump-diffusion models, International Journal of Theoretical and Applied Finance 14, 1005-1043.

Broadie, M. and Detemple, J. B.: 1996, American option valuation: New bounds, approximations, and a comparison of existing methods, The Review of Financial Studies 9, 1211-1250.

Cai, N.: 2009, On first passage times of a hyper-exponential jump diffusion process, Operations Research Letters 37, 127-134.

Cai, N.: 2011, Pricing and hedging quantile options in a flexible jump diffusion model, Journal of Applied Probability 48, 637-656.

Cai, N. and Kou, S.: 2011, Option pricing under a mixed-exponential jump diffusion model, Management Science 57, 2067-2081.

Cai, N. and Kou, S.: 2012, Pricing Asian options under a hyper-exponential jump-diffusion model, Operations Research 60, 64-77.

Carr, P.: 1998, Randomization and the American put, The Review of Financial Studies 11.

Carr, P., Geman, H., Madan, D. B. and Yor, M.: 2002, The fine structure of asset returns: An empirical investigation, Journal of Business 75, 305-332.

Carr, P. and Wu, L.: 2003, The finite moment log stable process and option pricing, The Journal of Finance 58, 753-778. 
Chiarella, C., Kang, B., Meyer, G. H. and Ziogas, A.: 2009, The evaluation of american option prices under stochastic volatility and jump-diffusion dynamics using the method of lines, International Journal of Theoretical and Applied Finance 12(03), 393-425.

Christoffersen, P.: 1998, Evaluating interval forecasts, International Economic Review pp. 841-862.

Crosby, J., Le Saux, N. and Mijatović, A.: 2010, Approximating lévy processes with a view to option pricing, International Journal of Theoretical and Applied Finance 13(01), 63-91.

Esscher, F.: 1932, On the probability function in the collective theory of risk., Skandinavisk Aktuarietidskrift 15, 175-195.

Fang, F. and Oosterlee, C. W.: 2008, A novel pricing method for european options based on fouriercosine series expansions, SIAM Journal on Scientific Computing 31(2), 826-848.

Gerber, H. U. and Shiu, E. S. W.: 1994, Option pricing by Esscher transforms, Transactions of the Society of Actuaries 46(99), 140.

Ingersoll, J. E.: 1996, Approximating american options and other financial contracts using barrier derivatives, Journal of Computational Finance 2, 85-112.

Jeannin, M. and Pistorius, M.: 2010, A transform approach to compute prices and Greeks of barrier options driven by a class of Lévy processes, Quantitative Finance 10(6), 629-644.

Kou, S. G.: 2002, A jump-diffusion model for option pricing, Management Science 48(8), 1086-1101.

Kou, S. G. and Wang, H.: 2003, First passage times of a jump diffusion process, Advances in Applied Probability 35(2), 504-531.

Kou, S. G. and Wang, H.: 2004, Option pricing under a double exponential jump diffusion model, Management Science 50(9), 1178-1192.

Kritzman, M. and Rich, D.: 2002, The mismeasurement of risk, Financial Analysts Journal 58(3), 9199.

Kupiec, P. H.: 1995, Techniques for verifying the accuracy of risk measurement models, The Journal of Derivatives $3(2), 73-84$. 
Kuznetsov, A.: 2013, On the convergence of the gaver-stehfest algorithm, SIAM Journal on Numerical Analysis 51(6), 2984-2998.

Leippold, M. and Vasiljević, N.: 2017, Pricing and disentanglement of american puts in the hyperexponential jump-diffusion model, Journal of Banking and Finance 77, 78-94.

Madan, D. B. and Seneta, E.: 1990, The variance gamma (VG) model for share market returns, Journal of Business 63(3), 511-524.

Merton, R. C.: 1976, Option pricing when underlying stock returns are discontinuous, Journal of Financial Economics 3(1-2), 125-144.

Monfort, A. and Pegoraro, F.: 2012, Asset pricing with second-order Esscher transforms, Journal of Banking and Finance 36(6), 1678-1687.

Ramezani, C. A. and Zeng, Y.: 2007, Maximum likelihood estimation of the double exponential jump-diffusion process, Annals of Finance 3(4), 487-507.

Rockafellar, R. T. and Uryasev, S.: 2002, Conditional value-at-risk for general loss distributions, Journal of Banking and Finance 7(26), 1443-1471.

Rossello, D.: 2008, MaxVaR with non-Gaussian distributed returns, European Journal of Operational Research 189(1), 159-171.

Sato, K.-I.: 1999, Lévy processes and infinitely divisible distributions, Cambridge University Press.

Sepp, A.: 2004, Analytical pricing of double-barrier options under a double-exponential jump diffusion process: applications of laplace transform, International Journal of Theoretical and Applied Finance 7(02), 151-175.

Steutel, F. W.: 1967, Note on the infinite divisibility of exponential mixtures, The Annals of Mathematical Statistics 38(4), 1303-1305.

Stulz, R.: 1996, Rethinking risk management, Journal of Applied Corporate Finance 9(3), 8-25. 
Thul, M. and Zhang, A. Q.: 2014, Analytical option pricing under an asymmetrically displaced double gamma jump-diffusion model, Working paper, 26th Australasian Finance and Banking Conference 2013.

Vuong, Q. H.: 1989, Likelihood ratio tests for model selection and non-nested hypotheses, Econometrica: Journal of the Econometric Society 57(2), 307-333. 


\section{A Properties of the D-MEM class}

The D-MEM in Definition 1 generalizes the mixed-exponential model (MEM) studied in Cai and Kou (2011) ${ }^{25}$ Indeed, with D-MEM we can approximate processes with completely monotone Lévy densities and jump-diffusion processes with arbitrary jump distributions. Therefore, several popular jump-diffusion models can be nested. For instance, we can interpret any MEM as the corresponding D-MEM without displacement $(\xi=0)$. Moreover, a zero displacement parameter also characterizes the class of hyper-exponential models (HEM), with the additional constraint that the mixing weights $\left\{p_{i}\right\}_{i=1, \ldots, m}$ and $\left\{q_{j}\right\}_{j=1, \ldots, n}$ in Equation (2) have to be strictly positive. Finally, double-exponential models (DEMs) represent a subclass of HEMs (with $n=m=1$ ), and therefore can also be included in the D-MEM family 26

\section{A.1 Measure change}

Given the inherent market incompleteness of Lévy models, we make use of the transform of Esscher (1932), which can be interpreted as a generalization of the Cameron-Martin-Girsanov change of measure ${ }^{27}$ Hence, we define the Radon-Nikodým derivative as

$$
Z_{t}(\vartheta):=\left.\frac{d \mathbb{Q}^{\vartheta}}{d \mathbb{P}}\right|_{\mathcal{F}_{t}}=\frac{e^{\vartheta X_{t}}}{\mathbb{E}\left[e^{\vartheta X_{t}}\right]},
$$

where $\mathbb{E}[\cdot]$ denotes the expectation under the physical probability measure $\mathbb{P}$. The process in (A.1) is well defined for an Esscher transform parameter $\vartheta \in\left(-\theta_{1}, \eta_{1}\right)$.

\footnotetext{
${ }^{25}$ MEMs are attractive because exponential mixtures are flexible enough to arbitrarily closely approximate any continuous function on $[0,+\infty)$. See, e.g., Botta and Harris (1986). Basically, the support for MEMs can be extended to the whole real line by considering separate approximations on the positive and the negative real line. This is especially convenient for symmetric functions because it is enough to approximate only half of the function. For example, Cai and Kou (2011), pp. 2077-2078, consider an MEM approximation of a normal distribution. However, this procedure is applicable only to normal distributions with zero mean or mode. In the case of a normal distribution with non-zero mean or mode, the two halves of the distribution have to be treated separately, and the procedure is computationally more expensive. In contrast, our model admits support in the form $[\mu,+\infty)$, with $\mu \in \mathbb{R},|\mu|<+\infty$.

${ }^{26}$ The HEM class of models was studied in Cai (2009, 2011), Crosby et al. (2010), Jeannin and Pistorius (2010), Boyarcheko and Boyarchenko (2011), Cai and Kou (2012), Leippold and Vasiljević (2017) among others. The main references for DEMs are Kou (2002), Kou and Wang (2003 |2004), Sepp (2004), AitSahlia and Runnemo (2007), Ramezani and Zeng (2007), Bayraktar and Xing (2009, 2011), Albrecher et al. (2012), among others.

${ }^{27}$ Gerber and Shiu (1994) proved that the Esscher transform approach can be justified within a rational expectations framework where the representative agent is characterized by a certain type of utility function. The main implication of this result for option pricing is that there exists a risk-neutral measure such that, in equilibrium, options are priced as expectations of their discounted payoffs.
} 
Proposition A.1 shows that the Esscher transform admits a structure-preserving change of measure for D-MEM processes. Moreover, the risk-neutral probability measure $\mathbb{Q}^{\vartheta}$ is uniquely identified by choosing the value of $\vartheta$ such that the forward no-arbitrage constraint is satisfied, i.e., the discounted process $\left\{e^{-r t} S_{t}, t \geq 0\right\}$ is a $\mathbb{Q}^{\vartheta}$-martingale.28

Proposition A.1 (Risk-neutral dynamics). The risk-neutral dynamics of the log-price process in the displaced mixed-exponential model (1)-(2) is given by

$$
X_{t}=X_{0}+\bar{r} t+\sigma W_{t}^{*}+\sum_{i=1}^{N_{t}^{*}} Y_{i}^{*}, \quad X_{0}:=\log S_{0}
$$

The processes $\left\{W_{t}^{*}, t \geq 0\right\}$ and $\left\{N_{t}^{*}, t \geq 0\right\}$ represent the Brownian motion and the Poisson process under the risk-neutral measure $\mathbb{Q}^{\vartheta}$, respectively. The compensated drift term is $\bar{r}:=r-\frac{\sigma^{2}}{2}-\lambda^{*} \zeta^{*}(1)$, where the parameter $r$ denotes the risk-free rate, parameter $\lambda^{*}$ is the jump intensity under the new measure, and function $\zeta^{*}(\cdot)$ is given by

$$
\zeta^{*}(u):=\left(\frac{\lambda_{+}^{*}}{\lambda^{*}} \sum_{i=1}^{m} \frac{p_{i}^{*} \eta_{i}^{*}}{\eta_{i}^{*}-u}+\frac{\lambda_{-}^{*}}{\lambda^{*}} \sum_{j=1}^{n} \frac{q_{j}^{*} \theta_{j}^{*}}{\theta_{j}^{*}+u}\right) e^{u \xi^{*}}-1 .
$$

The $\mathbb{Q}^{\vartheta}$-parameters can be computed as follows:

$$
\left\{\begin{array}{l}
\sigma^{*}=\sigma, \xi^{*}=\xi \\
\lambda_{+}^{*}=\lambda_{+} \sum_{i=1}^{m} \frac{p_{i} \eta_{i}}{\eta_{i}-\vartheta} e^{\vartheta \xi}, \quad \lambda_{-}^{*}=\lambda_{-} \sum_{j=1}^{n} \frac{q_{j} \theta_{j}}{\theta_{j}+\vartheta} e^{\vartheta \xi}, \lambda^{*}=\lambda_{+}^{*}+\lambda_{-}^{*}, \\
p_{i}^{*}=\frac{\frac{p_{i} \eta_{i}}{\eta_{i}-\vartheta}}{\sum_{i=1}^{m} \frac{p_{i} \eta_{i}}{\eta_{i}-\vartheta}}, \quad \eta_{i}^{*}=\eta_{i}-\vartheta, \quad \text { for } i=1,2, \ldots, m, \\
q_{j}^{*}=\frac{\frac{q_{j} \theta_{j}}{\theta_{j}+\vartheta}}{\sum_{j=1}^{n} \frac{q_{j} \theta_{j}}{\theta_{j}+\vartheta}}, \quad \theta_{j}^{*}=\theta_{j}+\vartheta, \quad \text { for } j=1,2, \ldots, n .
\end{array}\right.
$$

The Esscher transform parameter $\vartheta$ is the unique solution of the equation

$$
\mu-r-\lambda \zeta(1)+\vartheta \sigma^{2}+\Psi_{J}(\vartheta+1)-\Psi_{J}(\vartheta)=0
$$

where $\Psi_{J}(a)=\frac{1}{t} \log \mathbb{E}\left[e^{a \sum_{i=1}^{N_{t}} Y_{i}}\right]=\lambda \zeta(a)$ is the CGF of the jump part (i.e., the compound Poisson process).

\footnotetext{
${ }^{28}$ All proofs are relegated to Appendix $\mathrm{C}$
} 


\section{A.2 Pricing digital puts and first-passage disentanglement}

To calculate the digital puts $p\left(S_{t}, \tau ; K\right)$ and $P\left(S_{t}, \tau ; K\right)$ efficiently, we use the Laplace-Carson transform (LCT) approach. For any locally integrable function $f: \mathbb{R}^{+} \rightarrow \mathbb{R}$ and for all $\alpha \in \mathbb{R}^{+}$, the LCT is defined as

$$
(\mathscr{L} \mathscr{C})_{z}[f(z)](\alpha):=\tilde{f}(\alpha):=\alpha \int_{0}^{+\infty} e^{-\alpha z} f(z) d z .
$$

If we can calculate Canadized options in analytical form, we can use the Gaver-Stehfest inversion

algorithm to efficiently calculate the prices of American put options with finite maturity ${ }^{29}$ Propositions A.2 and A.3 present the Canadized European and one-touch digital puts, respectively. These results generalize the derivations in Leippold and Vasiljević (2017) to the D-MEM class.

Proposition A.2 (Canadized European digital put). Assume that the asset price process $\left\{S_{u}, t \leq u \leq T\right\}$ is described by the displaced mixed-exponential model (1). The price of a Canadized European digital put with the strike $K$ and the monitoring horizon $\tau$ is then given by

$$
\tilde{p}\left(S_{t}, \alpha ; K\right)= \begin{cases}1+\sum_{i=1}^{\hat{m}} \underline{w}_{i}\left(\frac{S_{t}}{K}\right)^{\beta_{i, \alpha}} & \text { if } S_{t}<K, \\ \sum_{j=1}^{\hat{n}} \bar{w}_{j}\left(\frac{S_{t}}{K}\right)^{\gamma_{j, \alpha}} & \text { if } S_{t} \geq K .\end{cases}
$$

The coefficients $\left\{\beta_{i, \alpha}\right\}_{i=1, \ldots, \hat{m}}$, and $\left\{\gamma_{j, \alpha}\right\}_{j=1, \ldots, \hat{n}}$ are the positive and negative roots of the characteristic equation (C.14). The number of positive and negative characteristic roots depends on the LCT parameter $\alpha$, i.e., $\hat{m}:=\hat{m}(\alpha) \leq m+1$ and $\hat{n}:=\hat{n}(\alpha) \leq n+1$, where $\hat{m}, \hat{n} \in \mathbb{N}$. The coefficients $\left\{\underline{w}_{i}\right\}_{i=1, \ldots, \hat{m}}$ and $\left\{\bar{w}_{j}\right\}_{j=1, \ldots, \hat{n}}$ can be computed by solving the system of linear equations

$$
\mathrm{Aw}=\mathbf{a} .
$$

The $(\hat{m}+\hat{n})$-dimensional column vector $\mathbf{w}$ and the $(m+n+2)$-dimensional column vector $\mathbf{a}$ are given in Equations (C.27) and (C.28), respectively. The matrix $\mathbf{A}$ is the $(m+n+2) \times(\hat{m}+\hat{n})$-dimensional matrix given in Equation (C.29).

Given the Canadized European digital put in analytical form, we can now apply the GaverStehfest inversion algorithm to obtain European digital put prices. It remains to find analytical

\footnotetext{
${ }^{29}$ We refer to, e.g., Abate and Whitt $(2006)$ and Kuznetsov (2013) for technical details about the Gaver-Stehfest inversion algorithm.
} 
expressions for the Canadized one-touch digital put.

Proposition A.3 (Canadized one-touch digital put). Assume that the asset price process $\left\{S_{u}, t \leq u \leq T\right\}$ is described by the displaced mixed-exponential model (1). The price of a Canadized one-touch digital put with the barrier level $K$ and the monitoring horizon $\tau$ is then given by

$$
\tilde{P}\left(S_{t}, \alpha ; K\right)= \begin{cases}\sum_{j=1}^{\hat{n}} v_{j}\left(\frac{S_{t}}{K}\right)^{\gamma_{j, \alpha}} & \text { if } S_{t}>K, \\ 1 & \text { if } S_{t} \leq K,\end{cases}
$$

using the same notation as in Proposition A.2. The set of coefficients $\left\{v_{j}\right\}_{j=1, \ldots, \hat{n}}$ satisfy the system of linear equations

$$
\mathbf{B v}=\mathbf{b}
$$

The $\hat{n}$-dimensional column vector $\mathbf{v}$ and the $(n+1)$-dimensional column vector $\mathbf{b}$ are given in Equations (C.40) and (C.41), respectively. The matrix $\mathbf{B}$ is the $(n+1) \times \hat{n}$-dimensional matrix given in Equation C.42.

Proposition A.4 extends the disentanglement results of Leippold and Vasiljević (2017) to the D-MEM class.

Proposition A.4 (Canadized first-passage disentanglement). In the D-MEM class, the price of a Canadized one-touch digital put with barrier $K$ and monitoring horizon $\tau$ can be decomposed as

$$
\tilde{P}\left(S_{t}, \alpha ; K\right)=\tilde{P}_{D}\left(S_{t}, \alpha ; K\right)+\tilde{P}_{J}\left(S_{t}, \alpha ; K\right),
$$

where $\tilde{P}_{D}(\cdot, \cdot ; \cdot)$ and $\tilde{P}_{J}(\cdot, \cdot ; \cdot)$, given by

$$
\begin{aligned}
& \tilde{P}_{D}\left(S_{t}, \alpha ; K\right)=\mathbb{1}_{\left\{S_{t}>K\right\}} \sum_{j=1}^{\hat{n}} \delta_{j}\left(\frac{S_{t}}{K}\right)^{\gamma_{j, \alpha}}+\mathbb{1}_{\left\{S_{t}=K\right\}}, \\
& \tilde{P}_{J}\left(S_{t}, \alpha ; K\right)=\mathbb{1}_{\left\{S_{t}>K\right\}} \sum_{j=1}^{\hat{n}} \iota_{j}\left(\frac{S_{t}}{K}\right)^{\gamma_{j, \alpha}}+\mathbb{1}_{\left\{S_{t}<K\right\}},
\end{aligned}
$$

represent the contribution of the diffusion process and of the jumps, respectively. The sets of coefficients $\left\{\delta_{j}\right\}_{j=1, \ldots, \hat{n}}$ and $\left\{\iota_{j}\right\}_{j=1, \ldots, \hat{n}}$ satisfy the systems of linear equations given in (C.49) and (C.51). 


\section{B D-MEM approximations of exponential Lévy processes}

In this section, we provide expressions for D-MEM approximations of several exponential Lévy models. They can be broadly classified into two groups: finite-activity jump-diffusion processes and completely monotone Lévy processes of infinite activity. In particular, we focus on the models that we consider in our empirical study, i.e., the MJD, FMLS, VG, and CGMY.

\section{B.1 Finite-activity jump-diffusion models: MJD}

As previously mentioned, DEMs, HEMs, and MEMs can be nested within the class of D-MEMs. This means that their respective D-MEM approximations are exact. On the other hand, the DMEM approximation is not so trivial for models with a "non-exponential" distribution of jump sizes. In particular, we consider the well known jump-diffusion model of Merton (1976). The log-price dynamics is described by Equation (1), and the jumps are assumed to be normally distributed, i.e., $Y \stackrel{\text { i.i.d. }}{\sim} \mathcal{N}\left(\mu_{J}, \sigma_{J}^{2}\right)$. Therefore, the Lévy density is given by

$$
\nu_{\mathrm{MJD}}(y)=\frac{\lambda}{\sqrt{2 \pi \sigma_{J}^{2}}} \exp \left(-\frac{\left(y-\mu_{J}\right)^{2}}{2 \sigma_{J}^{2}}\right),
$$

and the characteristic function is

$$
\begin{aligned}
\varphi_{\mathrm{MJD}}(u): & =\mathbb{E}\left[\exp \left(i u X_{t}\right)\right] \\
& =\exp \left(i u \mu t-\frac{u^{2} \sigma^{2} t}{2}+\lambda t\left(\exp \left(i u \mu_{J}-\frac{u^{2} \sigma_{J}^{2}}{2}\right)-1\right)\right) .
\end{aligned}
$$

Cai and Kou (2011), pp. 2077-2078, provide a MEM approximation of a normally distributed variable $Y \stackrel{\text { i.i.d. }}{\sim} \mathcal{N}\left(0,0.01^{2}\right)$. However, we pointed out in Appendix $\mathrm{A}$ that displaced mixed-exponential distributions are better suited for approximations of normal distributions with non-zero mean/mode. Using a simple change of variables, we now demonstrate that a D-MEM approximation of a normal distribution can be transformed into an MEM approximation.

Let us assume that the MEM approximation of the PDF of a normally distributed random variable $Y \stackrel{\text { i.i.d. }}{\sim} \mathcal{N}\left(0, \sigma_{J}^{2}\right)$ is given by

$$
f_{Y}(y) \approx 0.5 \sum_{i=1}^{m} p_{i} \eta_{i} e^{-\eta_{i}|y|}
$$


The PDF in Equation (B.2) is a special case of the general expressions for MEM and D-MEM probability distribution functions given in Equation (2). In addition to having zero displacement, the number of positive exponentials is equal to the number of negative exponentials $(m=n)$, and the parameters of the positive and the negative exponential functions and their corresponding mixing weights are component-wise identical, i.e., $\theta_{i}=\eta_{i}$ and $p_{i}=q_{i}$ for all $i=1,2, \ldots, m$, respectively. Furthermore, the mixture of exponential distributions is pre-multiplied by a constant which can be translated into the condition $\lambda_{+} / \lambda=\lambda_{-} / \lambda=0.5$, i.e., the activity rates of the positive and negative jumps are equal. The reason for all these restrictions on the MEM parameters is the symmetry property of normal distributions. Once the distribution of the random variable $Y$ is fitted with the desired accuracy, we can derive an approximation for any normally distributed variable $\tilde{Y} \stackrel{\text { i.i.d. }}{\sim} \mathcal{N}\left(\tilde{\mu}_{J}, \tilde{\sigma}_{J}^{2}\right)$. The two random variables can be expressed in terms of a standard normal variable $Z \stackrel{\text { i.i.d. }}{\sim} \mathcal{N}(0,1)$, i.e., $Y=\sigma_{J} Z$ and $\tilde{Y}=\tilde{\mu}_{J}+\tilde{\sigma}_{J} Z$. Therefore, we have that $\tilde{Y}=\tilde{\mu}_{J}+\frac{\tilde{\sigma}_{J}}{\sigma_{J}} Y$. Simple algebra gives us an expression for the D-MEM approximation for the distribution of $\mathcal{N}\left(\tilde{\mu}_{J}, \tilde{\sigma}_{J}^{2}\right)$ via the MEM approximation presented in Equation (B.2):

$$
f_{\tilde{Y}}(\tilde{y}) \approx 0.5 \sum_{i=1}^{m} \tilde{p}_{i} \tilde{\eta}_{i} e^{-\tilde{\eta}_{i}\left|\tilde{y}-\tilde{\mu}_{J}\right|},
$$

with $\tilde{\eta}_{i}=\frac{\sigma_{J}}{\tilde{\sigma}_{J}} \eta_{i}$ and $\tilde{p}_{i}=p_{i}$ for $i=1,2, \ldots, m$. As expected, the displacement parameter is $\xi=\tilde{\mu}_{J}$. Finally, from the perspective of Merton's jump-diffusion model, the diffusion parameter $\sigma$ and the jump intensity $\lambda$ remain unchanged, which is justified by the fact that the D-MEM approximation in Equation (B.3) affects only the distribution of the jump sizes.

\section{B.2 Completely monotone Lévy processes: FMLS, VG, and CGMY}

All the pure-jump Lévy processes studied in this paper, namely, the FMLS, VG, and CGMY, are completely monotone. It is precisely this property that allows us to approximate the considered Lévy processes by hyper-exponential models. In particular, a Lévy density $\nu:(0,+\infty) \rightarrow \mathbb{R}$ is said to be completely monotone if for all $k \in \mathbb{N}_{0}^{+}$it is of class $\mathcal{C}^{\infty}$ and if $(-1)^{k} d^{k} \nu(y) / d y^{k}>0$ for all $y>0$; see, e.g., Sato (1999), p. 388 ${ }^{30}$ Furthermore, Bernstein's theorem ensures that a Lévy density is

\footnotetext{
${ }^{30}$ This definition can be easily extended to the whole real line. The condition for complete monotonicity then becomes $(-1)^{k} d^{k} \nu(|y|) / d y^{k}>0$ for $y \in(-\infty,+\infty)$.
} 
completely monotone if and only if it can be decomposed as

$$
\nu(y)=\mathbb{1}_{\{y<0\}} \int_{-\infty}^{0} e^{-v y} \rho_{-}(d v)+\mathbb{1}_{\{y>0\}} \int_{0}^{+\infty} e^{-v y} \rho_{+}(d v),
$$

where $\rho_{-}(d v)$ and $\rho_{+}(d v)$ are Radon measures on the intervals $(-\infty, 0)$ and $(0,+\infty)$, respectively, so that the two integrals are finite. In a nutshell, this means that the arrival rate of the jumps is decreasing with the jump size. If the integrals in Equation (B.4) are discretized, the completely monotone Lévy density $\nu(\cdot)$ is approximated by a finite mixture of exponential densities. Moreover, jumps with an expected size smaller than a certain threshold, i.e., jumps with magnitudes close to zero, can be approximated by a diffusion process. Based on this approximation, Asmussen et al. (2007) infer that completely monotone jump models can be approximated by hyper-exponential jump-diffusion models, which are a subset of the displaced mixed-exponential class.

As an example of the hyper-exponential approximation of a completely monotone Lévy model, we consider the CGMY process of Carr et al. (2002). It is a pure-jump process, and its Lévy density is given by the exponentially dampened power law

$$
\nu_{C G M Y}(y)=C\left(\frac{e^{-G|y|}}{|y|^{1+Y}} \mathbb{1}_{\{y<0\}}+\frac{e^{-M y}}{y^{1+Y}} \mathbb{1}_{\{y>0\}}\right),
$$

and its characteristic function is

$$
\begin{aligned}
\varphi_{\mathrm{CGMY}}(u): & =\mathbb{E}\left[\exp \left(i u X_{\mathrm{CGMY}}(t)\right)\right] \\
& =\exp \left(i u \omega t+t C \Gamma(-Y)\left((M-i u)^{Y}-M^{Y}+(G+i u)^{Y}-G^{Y}\right)\right),
\end{aligned}
$$

where $\omega:=-C \Gamma(-Y)\left((M-1)^{Y}-M^{Y}+(G+1)^{Y}-G^{Y}\right)$ represents the convexity adjustment, and $\Gamma(\cdot)$ denotes the (mathematical) Gamma function. The parameter $C \in \mathbb{R}^{+}$represents the jump intensity. The parameters $G \in \mathbb{R}_{0}^{+}$and $M \in \mathbb{R}_{0}^{+}$are exponential decay parameters for the negative and positive jumps, respectively. Depending on their relative values, the model can generate positive, negative, or zero skewness. The parameter $Y$ is especially interesting because it characterizes the so called "fine structure" of asset returns, i.e., it describes the behavior of the Lévy density in the neighborhood of zero ${ }^{31}$ Following the idea of Asmussen et al. (2007), section 2.1, pp. 85-87, and

\footnotetext{
${ }^{31} \mathrm{~A}$ CGMY process is completely monotone if $Y \in(-1,2)$. For $Y<0$ the process is of finite activity, for $Y \in(0,1)$ it is characterized by infinite activity and finite variation, and for $Y \in(1,2)$ it has infinite variation but finite quadratic variation.
} 
Jeannin and Pistorius (2010), section 2, pp. 631-632, our starting point is the identity

$$
\frac{1}{y^{1+Y}}=\frac{1}{\Gamma(1+Y)} \int_{0}^{+\infty} u^{Y} e^{-u y} d u
$$

which holds for all $Y \in \mathbb{C} \backslash\{-2,-3,-4, \ldots\}$. This expression follows directly from the definition of the Gamma function. Discretization of the integral in Equation (B.7) yields

$$
\frac{1}{y^{1+Y}} \approx \frac{1}{\Gamma(1+Y)} \sum_{i=1}^{N-1} u_{i}^{Y} e^{-u_{i} y}\left(u_{i+1}-u_{i}\right) .
$$

The partition $\Pi(N):=\left(u_{i}\right)_{i=1,2, \ldots, N-1}$ of the interval $(0,+\infty)$ is such that $\Delta_{\Pi(N)} \rightarrow 0$ when $N \rightarrow \infty$, with the norm defined as $\Delta_{\Pi(N)}=\max _{1 \leq i \leq N-1}\left|u_{i+1}-u_{i}\right|$. Therefore, a completely monotone process can be approximated with arbitrary accuracy by choosing the appropriate partition of the integration interval. For example, the CGMY density (B.5) can be approximated by

$$
\begin{aligned}
\nu_{C G M Y}(y) & \approx \frac{C}{\Gamma(1+Y)} \sum_{i=1}^{N^{+}-1} w_{i}^{+} e^{-\left(M+u_{i}\right) y_{1}} \mathbb{1}_{\{y>0\}} \\
& +\frac{C}{\Gamma(1+Y)} \sum_{j=1}^{N^{-}-1} w_{j}^{-} e^{-\left(G+v_{j}\right)|y|} \mathbb{1}_{\{y<0\}},
\end{aligned}
$$

where $w_{i}^{+}:=u_{i}^{Y}\left(u_{i+1}-u_{i}\right)$ for $i=1,2, \ldots, N^{+}$, and $w_{j}^{-}:=v_{j}^{Y}\left(v_{j+1}-v_{j}\right)$ for $j=1,2, \ldots, N^{-}$. We implicitly introduced the partition $U\left(N^{+}\right):=\left(u_{i}\right)_{i=1,2, \ldots, N^{+}-1}$ of the interval $(0,+\infty)$, as well as the partition $V\left(N^{-}\right):=\left(v_{j}\right)_{j=1,2, \ldots, N^{-}-1}$ of the interval $(-\infty, 0)$. One can easily check that Equation (B.9) corresponds to the D-MEM Lévy density (2) with the following parameters: $m=N^{+}-1$, $n=N^{-}-1, \eta_{i}=M+u_{i}$ and $p_{i}=w_{i}^{+} / \eta_{i}$ for $i=1,2, \ldots, m, \theta_{j}=G+v_{j}$ and $q_{j}=w_{j}^{-} / \theta_{j}$ for $j=1,2, \ldots, n, \lambda_{+}=\tilde{C} \sum_{i=1}^{N^{+}-1} p_{i}$ and $\lambda_{-}=\tilde{C} \sum_{j=1}^{N^{-}-1} q_{j}$, where $\tilde{C}=C / \Gamma(1+Y)$ Therefore, depending on the discretization and the truncation error, which are determined by the choice of the partition of the positive and negative semi-axes, we can obtain an arbitrarily accurate approximation of the CGMY density.

In practical applications, Asmussen et al. (2007) fix in advance the number of components in the mixture as well as their exponential decay parameters. Subsequently, they minimize the distance between the two Lévy densities by optimally choosing the partition of the integration intervals. Jeannin and Pistorius (2010) follow an almost identical procedure, except that they optimize the

\footnotetext{
${ }^{32}$ We note that in the D-MEM approximation of a CGMY process, the displacement parameter is equal to zero. More generally, the displacement has to be zero for any model with infinite activity: otherwise, the integral of the Lévy density would not exist.
} 
mixing weights 33 Nevertheless, the approximation derived in Equation (B.9) implies that there exists a structural relation between the exponential decay parameters and the corresponding mixing weights. Hence, the total number of jumps and the values of the model parameters are in fact determined by the chosen partition. Consistently with the theoretical results presented in Equations (B.7) - B.9), and simultaneously avoiding a computationally burdensome numerical optimization without loss of accuracy, we make a trade-off by choosing a larger number of exponential terms. ${ }^{34}$ However, for the remaining steps in our exercise, e.g., for the approximation of small jumps by a diffusion, we closely follow the procedure outlined in Asmussen et al. (2007) and Jeannin and Pistorius (2010) since it ensures the weak convergence of the constructed sequence of HEM processes to the target Lévy process.

In addition to the CGMY model, we consider two other popular pure-jump models: the VG model of Madan and Seneta (1990) and the FMLS model of Carr and Wu (2003). Both models are special cases of the CGMY. The Lévy density of a VG process is obtained by setting the fine structure parameter to zero in Equation (B.5). On the other hand, the FMLS process is a spectrally negative Lévy process, i.e., positive jumps are completely excluded, and the exponential decay parameter of the negative jumps is set to zero. We do not give explicitly the Lévy measures and the characteristic functions of the VG and FMLS processes because they can be inferred directly from Equation (B.5) using the aforementioned restrictions.

\footnotetext{
${ }^{33}$ The number of exponential terms in the mixture varies between five and seven in Asmussen et al. (2007) and Jeannin and Pistorius (2010).

${ }^{54}$ In particular, we use 50 (non-degenerate) exponential terms in the mixture. Our numerical tests show that the suggested procedure is generally fast and stable. It is important to stress that a detailed investigation of the three approximation approaches described in this section is a separate research topic. The algorithms outlined here are by no means the major concern of our paper, and the sole purpose of this section is to briefly describe our approach and relate it to the relevant literature. Admittedly, there are also other approaches in the literature, e.g., Crosby et al. (2010). Nonetheless, we find that our modification of Asmussen et al. (2007) and Jeannin and Pistorius (2010) works sufficiently well for our application.
} 


\section{Proofs}

\section{C.1 Proof of Proposition A.1: Risk-neutral dynamics}

The Esscher transform of a D-MEM process is

$$
Z_{t}(\vartheta):=\left.\frac{d \mathbb{Q}^{\vartheta}}{d \mathbb{P}}\right|_{\mathcal{F}_{t}}=\underbrace{e^{\vartheta \sigma W_{t}-\frac{1}{2} \vartheta^{2} \sigma^{2} t}}_{Z_{t}^{D}(\vartheta)} \underbrace{e^{\vartheta \sum_{i=1}^{N_{t}} Y_{i}-t \Psi_{J}(\vartheta)}}_{Z_{t}^{J}(\vartheta)} .
$$

First, it follows from the Girsanov theorem that the $\mathbb{Q}^{\vartheta}$-Brownian motion is $W_{t}^{*}=W_{t}-[W, \vartheta \sigma W]_{t}=$ $W_{t}-\vartheta \sigma t$. The volatility parameter remains the same after the change of measure, i.e., $\sigma^{*}=\sigma$. Second, it can be shown that the cumulant generating function (CGF) of the jump part is given by

$$
\Psi_{J}(a)=\lambda\left(\left(\frac{\lambda_{+}}{\lambda} \sum_{i=1}^{m} \frac{p_{i} \eta_{i}}{\eta_{i}-a}+\frac{\lambda_{-}}{\lambda} \sum_{j=1}^{n} \frac{q_{j} \theta_{j}}{\theta_{j}+a}\right) e^{a \xi}-1\right),
$$

for every $a \in\left(-\theta_{1}, \eta_{1}\right)$. The jump CGF under the new measure is

$$
\Psi_{J}^{*}(\varkappa):=\frac{1}{t} \log \mathbb{E}\left[Z_{t}^{J}(\vartheta) e^{\varkappa \sum_{i=1}^{N_{t}} Y_{i}}\right]=\Psi_{J}(\varkappa+\vartheta)-\Psi_{J}(\vartheta)
$$

for every $\varkappa \in\left(-\theta_{1}, \eta_{1}\right)$. This equation can be written in the form

$$
\begin{aligned}
\Psi_{J}^{*}(\varkappa) & =\lambda \int_{-\infty}^{+\infty}\left(e^{\varkappa y}-1\right) e^{\vartheta y} f_{Y}(y) d y \\
& =\lambda^{*} \int_{-\infty}^{+\infty}\left(e^{\varkappa y}-1\right) \frac{e^{\vartheta y} f_{Y}(y)}{\int_{-\infty}^{+\infty} e^{\vartheta y} f_{Y}(y) d y} d y
\end{aligned}
$$

where the jump intensity under the new measure is given by

$$
\lambda^{*}:=\lambda \int_{-\infty}^{+\infty} e^{\vartheta y} f_{Y}(y) d y=\underbrace{\lambda_{+} \sum_{i=1}^{m} \frac{p_{i} \eta_{i}}{\eta_{i}-\vartheta} e^{\vartheta \xi}}_{\lambda_{+}^{*}}+\underbrace{\lambda_{-} \sum_{j=1}^{n} \frac{q_{j} \theta_{j}}{\theta_{j}+\vartheta} e^{\vartheta \xi}}_{\lambda_{-}^{*}},
$$

and the exponentially tilted jump size distribution under the new measure is defined as

$$
f_{Y}^{*}(y):=\frac{e^{\vartheta y} f_{Y}(y)}{\int_{-\infty}^{+\infty} e^{\vartheta y} f_{Y}(y) d y} .
$$

After some algebraic calculation, we obtain the expression for the jump CGF under the risk-neutral measure

$$
\Psi_{J}^{*}(\varkappa)=\lambda^{*}\left(\left(\frac{\lambda_{+}^{*}}{\lambda^{*}} \sum_{i=1}^{m} \frac{p_{i}^{*} \eta_{i}^{*}}{\eta_{i}^{*}-\varkappa}+\frac{\lambda_{-}^{*}}{\lambda^{*}} \sum_{j=1}^{n} \frac{q_{j}^{*} \theta_{j}^{*}}{\theta_{j}^{*}+\varkappa}\right) e^{\varkappa \xi^{*}}-1\right)=: \lambda^{*} \zeta^{*}(\varkappa) .
$$


The coefficients $\lambda_{+}^{*}$ and $\lambda_{-}^{*}$ are defined in Equation C.5. Furthermore, for all $i=1,2, \ldots, m$ we have that $\eta_{i}^{*}=\eta_{i}-\vartheta$ and $p_{i}^{*}=p_{i} \frac{\eta_{i}}{\eta_{i}-\vartheta} \frac{1}{v_{+}(\vartheta)}$, and for all $j=1,2, \ldots, n$ the adjusted coefficients are $\theta_{j}^{*}=\theta_{j}+\vartheta$ and $q_{j}^{*}=q_{j} \frac{\theta_{j}}{\theta_{j}+\vartheta} \frac{1}{v_{-}(\vartheta)}$. The introduced $v$-coefficients are $v_{+}(\vartheta)=\sum_{i=1}^{m} \frac{p_{i} \eta_{i}}{\eta_{i}-\vartheta}$ and $v_{-}(\vartheta)=\sum_{j=1}^{n} \frac{q_{j} \theta_{j}}{\theta_{j}+\vartheta}$. The displacement parameter $\xi$ remain unchanged, i.e., $\xi^{*}=\xi$. Thus, we conclude that the log-price process under the measure $\mathbb{Q}^{\vartheta}$ is described by Equation A.2.

It remains to find the value of the Esscher transform parameter $\vartheta$ which guarantees that the new probability measure is indeed the risk-neutral measure. We require that the discounted process $\left\{e^{-r t} S_{t}, t \geq 0\right\}$ is a $\mathbb{Q}^{\vartheta}$-martingale, i.e.,

$$
\mathbb{E}^{*}\left[e^{-r t+X_{t}}\right]=e^{(\bar{\mu}-r) t} e^{\frac{1}{2}(2 \vartheta+1) \sigma^{2} t} e^{\left(\Psi_{J}(\vartheta+1)-\Psi_{J}(\vartheta)\right) t}=1
$$

Therefore, our equivalent martingale measure $\mathbb{Q}^{\vartheta}$ is indeed the risk-neutral measure if the Esscher transform parameter satisfies the equation

$$
\bar{\mu}-r+\left(\vartheta+\frac{1}{2}\right) \sigma^{2}+\Psi_{J}(\vartheta+1)-\Psi_{J}(\vartheta)=0,
$$

where $\bar{\mu}:=\mu-\lambda \zeta(1)-\frac{\sigma^{2}}{2}$ represents the compensated drift term. It can be easily verified that this equation can be reduced to the form (A.4). The proof of existence and uniqueness of $\vartheta$ for the change of measure via the Esscher transform is given in Thul and Zhang (2014), proposition 2, pp. 12-13.

\section{C.2 Proof of Proposition A.2: The pricing of Canadized European digital puts in the D-MEM framework}

First, for computational convenience, we recast the original problem in the log-space, i.e., we define the $\log$-price $x:=\log S_{t}$ and the $\log$-strike $\kappa:=\log K$. Second, we note that the Feynman-Kac theorem allows us to compute the European digital option price in Equation (10) by solving the partial integro-differential equation (PIDE)

$$
-\frac{\partial p}{\partial \tau}(x, \tau)+\frac{\sigma^{2}}{2} \frac{\partial^{2} p}{\partial x^{2}}(x, \tau)+\bar{\mu} \frac{\partial p}{\partial x}(x, \tau)+\int_{-\infty}^{+\infty}[p(x+y, \tau)-p(x, \tau)] \nu(y) d y=0,
$$

where $\bar{\mu}$ denotes the compensated drift term of the log-price process under the physical measure, and the Lévy measure $\nu(\cdot)$ is defined in Equation (2). The boundary and initial conditions are: 
$\lim _{x \uparrow+\infty} p(x, \tau)=0, \lim _{x \downarrow-\infty} p(x, \tau)=1$, and $\lim _{\tau \downarrow 0} p(x, \tau)=\mathbb{1}_{\{x<\kappa\}}$.

To solve this equation, we first introduce the change of variables $y \mapsto y^{\prime}:=y-\xi$. Therefore, the transformed jump distribution takes the form

$$
f_{Y}\left(y^{\prime}\right)=\frac{\lambda_{+}}{\lambda} \sum_{i=1}^{m} p_{i} \eta_{i} e^{-\eta_{i} y^{\prime}} \mathbb{1}_{\left\{y^{\prime} \geq 0\right\}}+\frac{\lambda_{-}}{\lambda} \sum_{j=1}^{n} q_{j} \theta_{j} e^{\theta_{j} y^{\prime}} \mathbb{1}_{\left\{y^{\prime}<0\right\}} .
$$

Third, taking the Laplace-Carson transform (LCT) of the PIDE (C.10), we obtain the ordinary integro-differential equation (OIDE) for the Canadized European digital put $\tilde{p}:=\tilde{p}(x, \alpha)$ :

$$
\frac{\sigma^{2}}{2} \frac{d^{2} \tilde{p}}{d x^{2}}(x, \alpha)+\bar{\mu} \frac{d \tilde{p}}{d x}(x, \alpha)-(\lambda+\alpha) \tilde{p}(x, \alpha)+\alpha \mathbb{1}_{\{x<\kappa\}}+\lambda \int_{-\infty}^{+\infty} \tilde{p}\left(x+\xi+y^{\prime}, \alpha\right) f_{Y}\left(y^{\prime}\right) d y^{\prime}=0 .
$$

The initial condition is absorbed into Equation C.12 due to the LCT. On the other hand, the transformed boundary conditions are: $\lim _{x \downarrow-\infty} \tilde{p}(x, \alpha)=1$ and $\lim _{x \uparrow+\infty} \tilde{p}(x, \alpha)=0$.

We conjecture a solution in the form

$$
\tilde{p}(x, \alpha)= \begin{cases}1+\sum_{l=1}^{\hat{m}} \underline{w}_{l}^{\prime} e^{\beta_{l, \alpha}(x-\kappa-\xi)} & \text { if } x<\kappa, \\ \sum_{l=1}^{\hat{n}} \bar{w}_{l}^{\prime} e^{\gamma_{l, \alpha}(x-\kappa-\xi)} & \text { if } x \geq \kappa .\end{cases}
$$

The coefficients $\left\{\beta_{i, \alpha}\right\}_{i=1, \ldots, \hat{m}}$ and $\left\{\gamma_{j, \alpha}\right\}_{j=1, \ldots, \hat{n}}$ represent the positive and negative roots, respectively, of the characteristic equation

$$
\Psi^{\prime}(u)=\alpha, \alpha \in \mathbb{R}^{+}
$$

where $\Psi^{\prime}(u):=\bar{\mu} u+\frac{1}{2} \sigma^{2} u^{2}+\lambda\left(\frac{\lambda_{+}}{\lambda} \sum_{i=1}^{m} \frac{p_{i} \eta_{i}}{\eta_{i}-u}+\frac{\lambda_{-}}{\lambda} \sum_{j=1}^{n} \frac{q_{j} \theta_{j}}{\theta_{j}+u}-1\right) 36$ The roots, which can be computed numerically, satisfy the ordering relation

$$
-\infty<\gamma_{\hat{n}, \alpha}<\cdots<\gamma_{2, \alpha}<\gamma_{1, \alpha}<0<\beta_{1, \alpha}<\beta_{2, \alpha}<\cdots<\beta_{\hat{m}, \alpha}<+\infty
$$

where $\hat{m}:=\hat{m}(\alpha) \leq m+1$ and $\hat{n}:=\hat{n}(\alpha) \leq n+1$. The coefficients $\left\{\underline{w}_{i}\right\}_{i=1, \ldots, \hat{m}}$ and $\left\{\bar{w}_{j}\right\}_{j=1, \ldots, \hat{n}}$ can be calculated by analyzing the solution in the two different regions, i.e., below and above the strike price, respectively.

\footnotetext{
${ }^{35}$ We note that the Equation C.13 is equivalent to the Equation A.6). The difference is that the former represents a "displaced" version of the latter (i.e., the displacement parameter $\xi$ is explicitly included in the exponential terms, and we defined $\underline{w}_{l}^{\prime}:=\underline{w}_{l} e^{\beta_{l, \alpha} \xi}$ for $l=1, \cdots, \hat{m}$ and $\bar{w}_{l}^{\prime}:=\bar{w}_{l} e^{\gamma_{l, \alpha} \xi}$ for $\left.l=1, \cdots, \hat{n}\right)$. The introduction of expression C.13 is a merely formal and intermediate step, however it is necessary to analytically solve the OIDE (C.12).

${ }^{36}$ It can be verified that $\Psi^{\prime}(u)=\left.\Psi(u)\right|_{\xi=0}+\lambda\left(\frac{\lambda_{+}}{\lambda} \sum_{i=1}^{m} \frac{p_{i} \eta_{i}}{\eta_{i}-1}+\frac{\lambda_{-}}{\lambda} \sum_{j=1}^{n} \frac{q_{j} \theta_{j}}{\theta_{j}+1}\right)\left(1-e^{\xi}\right)$.
} 
First, we consider the case $x<\kappa$. It is straightforward to compute the two derivative terms in Equation (C.12), i.e.,

$$
\begin{aligned}
& \frac{d \tilde{p}}{d x}(x, \alpha)=\sum_{l=1}^{\hat{m}} \underline{w}_{l}^{\prime} \beta_{l, \alpha} e^{\beta_{l, \alpha}(x-\kappa-\xi)} \\
& \frac{d^{2} \tilde{p}}{d x^{2}}(x, \alpha)=\sum_{l=1}^{\hat{m}} \underline{w}_{l}^{\prime} \beta_{l, \alpha}^{2} e^{\beta_{l, \alpha}(x-\kappa-\xi)} .
\end{aligned}
$$

On the other hand, the integral term $\mathscr{I}:=\int_{-\infty}^{+\infty} \tilde{p}\left(x+\xi+y^{\prime}, \alpha\right) f_{Y}\left(y^{\prime}\right) d y^{\prime}$ is much more involved. It can be decomposed as

$$
\begin{aligned}
\mathscr{I} & =\frac{\lambda_{-}}{\lambda} \sum_{l=1}^{\hat{m}} \sum_{j=1}^{n} \int_{-\infty}^{0} q_{j} \theta_{j} \underline{w}_{l}^{\prime} e^{\beta_{l, \alpha}(x-\kappa)} e^{\left(\beta_{l, \alpha}+\theta_{j}\right) y^{\prime}} d y^{\prime} \\
& +\frac{\lambda_{-}}{\lambda} \sum_{j=1}^{n} \int_{-\infty}^{0} q_{j} \theta_{j} e^{\theta_{j} y^{\prime}} d y^{\prime} \\
& +\frac{\lambda_{+}}{\lambda} \sum_{l=1}^{\hat{m}} \sum_{i=1}^{m} \int_{0}^{\kappa-x} p_{i} \eta_{i} \underline{w}_{l}^{\prime} e^{\beta_{l, \alpha}(x-\kappa)} e^{\left(\beta_{l, \alpha}-\eta_{i}\right) y^{\prime}} d y^{\prime} \\
& +\frac{\lambda_{+}}{\lambda} \sum_{l=1}^{m} \int_{0}^{\kappa-x} p_{l} \eta_{l} e^{-\eta_{l} y^{\prime}} d y^{\prime} \\
& +\frac{\lambda_{+}}{\lambda} \sum_{i=1}^{m} \sum_{l=1}^{\hat{n}} \int_{\kappa-x}^{+\infty} p_{i} \eta_{i} \bar{w}_{l}^{\prime} e^{\gamma_{l, \alpha}(x-\kappa)} e^{\left(\gamma_{l, \alpha}-\eta_{i}\right) y^{\prime}} d y^{\prime} .
\end{aligned}
$$

After some algebra, the OIDE C.12 yields the condition

$$
\sum_{l=1}^{\hat{m}} \underline{w}_{l} e^{\beta_{l, \alpha}(x-\kappa)}\left(\Psi^{\prime}\left(\beta_{l, \alpha}\right)-\alpha\right)-\sum_{l=1}^{m} \lambda_{+} p_{l} \eta_{l} e^{\eta_{l}(x-\kappa)}\left(\sum_{i=1}^{\hat{m}} \frac{\underline{w}_{i}}{\eta_{l}-\beta_{i, \alpha}}-\sum_{j=1}^{\hat{n}} \frac{\bar{w}_{j}}{\eta_{l}-\gamma_{j, \alpha}}+\frac{1}{\eta_{l}}\right)=0,
$$

Using the characteristic equation (C.14), we conclude that the first sum in Equation (C.18) is equal to zero. Therefore, we obtain a system of $m$ linear equations for the coefficients $\left\{\underline{w}_{i}\right\}_{i=1, \ldots, \hat{m}}$ and $\left\{\bar{w}_{j}\right\}_{j=1, \ldots, \hat{n}}:$

$$
\sum_{i=1}^{\hat{m}} \frac{\underline{w}_{i}}{\eta_{l}-\beta_{i, \alpha}}-\sum_{j=1}^{\hat{n}} \frac{\bar{w}_{j}}{\eta_{l}-\gamma_{j, \alpha}}=-\frac{1}{\eta_{l}}, \text { for } l=1, \ldots, m
$$

Now we study the case $x \geq \kappa$. First, the derivative terms are given by

$$
\begin{aligned}
& \frac{d \tilde{p}}{d x}(x, \alpha)=\sum_{l=1}^{\hat{n}} \bar{w}_{l}^{\prime} \gamma_{l, \alpha} e^{\gamma_{l, \alpha}(x-\kappa-\xi)} \\
& \frac{d^{2} \tilde{p}}{d x^{2}}(x, \alpha)=\sum_{l=1}^{\hat{n}} \bar{w}_{l}^{\prime} \gamma_{l, \alpha}^{2} e^{\gamma_{l, \alpha}(x-\kappa-\xi)}
\end{aligned}
$$

The integral term can be decomposed as

$$
\begin{aligned}
\mathscr{I} & =\frac{\lambda_{-}}{\lambda} \sum_{j=1}^{n} \sum_{l=1}^{\hat{m}} \int_{-\infty}^{\kappa-x} q_{j} \theta_{j} \underline{w}_{l}^{\prime} e^{\beta_{l, \alpha}(x-\kappa)} e^{\left(\beta_{l, \alpha}+\theta_{j}\right) y^{\prime}} d y^{\prime} \\
& +\frac{\lambda_{-}}{\lambda} \sum_{j=1}^{n} \int_{-\infty}^{\kappa-x} q_{j} \theta_{j} e^{\theta_{j} y^{\prime}} d y^{\prime} \\
& +\frac{\lambda_{-}}{\lambda} \sum_{l=1}^{\hat{n}} \sum_{j=1}^{n} \int_{\kappa-x}^{0} q_{j} \theta_{j} \bar{w}_{l}^{\prime} e^{\gamma_{l, \alpha}(x-\kappa)} e^{\left(\gamma_{l, \alpha}+\theta_{j}\right) y^{\prime}} d y^{\prime} \\
& +\frac{\lambda_{+}}{\lambda} \sum_{l=1}^{\hat{n}} \sum_{i=1}^{m} \int_{0}^{+\infty} p_{i} \eta_{i} \bar{w}_{l}^{\prime} e^{\gamma_{l, \alpha}(x-\kappa)} e^{\left(\gamma_{l, \alpha}-\eta_{i}\right) y^{\prime}} d y^{\prime} .
\end{aligned}
$$


Again, after some lengthy calculations we obtain the condition

$$
\sum_{l=1}^{\hat{n}} \bar{w}_{l} e^{\gamma_{l, \alpha}(x-\kappa)}\left(\Psi^{\prime}\left(\gamma_{l, \alpha}\right)-\alpha\right)+\sum_{l=1}^{n} \lambda_{-} q_{l} \theta_{l} e^{\theta_{l}(x-\kappa)}\left(\sum_{i=1}^{\hat{m}} \frac{\underline{w}_{i}}{\theta_{l}+\beta_{i, \alpha}}-\sum_{j=1}^{\hat{n}} \frac{\bar{w}_{j}}{\theta_{l}+\gamma_{j, \alpha}}+\frac{1}{\theta_{l}}\right)=0 .
$$

Using the same arguments as in the case $x<\kappa$, we get the following set of linear equations:

$$
\sum_{i=1}^{\hat{m}} \frac{\underline{w}_{i}}{\theta_{l}+\beta_{i, \alpha}}-\sum_{j=1}^{\hat{n}} \frac{\bar{w}_{j}}{\theta_{l}+\gamma_{j, \alpha}}=-\frac{1}{\theta_{l}}, \text { for } l=1, \ldots, n
$$

To close the system of equations we use the value matching and the smooth pasting conditions at $x=\kappa:$

$$
\begin{aligned}
& \lim _{x \uparrow \kappa} \tilde{p}(x, \alpha)=\lim _{x \downarrow \kappa} \tilde{p}(x, \alpha), \\
& \lim _{x \uparrow \kappa} \frac{d \tilde{p}}{d x}(x, \alpha)=\lim _{x \downarrow \kappa} \frac{d \tilde{p}}{d x}(x, \alpha) .
\end{aligned}
$$

Therefore, we have

$$
\begin{array}{ll}
\sum_{i=1}^{\hat{m}} \underline{w}_{i}-\sum_{j=1}^{\hat{n}} \bar{w}_{j} & =-1, \\
\sum_{i=1}^{\hat{m}} \beta_{i, \alpha} \underline{w}_{i}-\sum_{j=1}^{\hat{n}} \gamma_{j, \alpha} \bar{w}_{j} & =0 .
\end{array}
$$

After collecting Equations (C.19, C.23, and C.25 we obtain the following system of linear equations:

$$
\mathbf{A w}=\mathbf{a}
$$

where

$$
\mathbf{w}=\left(\underline{w}_{1}, \ldots, \underline{w}_{\hat{m}}, \bar{w}_{1}, \ldots, \bar{w}_{\hat{n}}\right)^{\prime}
$$

is an $(\hat{n}+\hat{m})$-dimensional column vector, and

$$
\mathbf{a}=\left(-1,0,-\frac{1}{\eta_{1}}, \ldots,-\frac{1}{\eta_{m}},-\frac{1}{\theta_{1}}, \ldots,-\frac{1}{\theta_{n}}\right)^{\prime}
$$


is an $(m+n+2)$-dimensional column vector. Lastly, the matrix $\mathbf{A}$ is given by

$$
\mathbf{A}=\left(\begin{array}{cccccc}
1 & \cdots & 1 & -1 & \cdots & -1 \\
\beta_{1, \alpha} & \cdots & \beta_{\hat{m}, \alpha} & -\gamma_{1, \alpha} & \cdots & -\gamma_{\hat{n}, \alpha} \\
\frac{1}{\eta_{1}-\beta_{1, \alpha}} & \cdots & \frac{1}{\eta_{1}-\beta_{\hat{m}, \alpha}} & -\frac{1}{\eta_{1}-\gamma_{1, \alpha}} & \cdots & -\frac{1}{\eta_{1}-\gamma_{\hat{n}, \alpha}} \\
\vdots & \ddots & \vdots & \vdots & \ddots & \vdots \\
\frac{1}{\eta_{m}-\beta_{1, \alpha}} & \cdots & \frac{1}{\eta_{m}-\beta_{\hat{m}, \alpha}} & -\frac{1}{\eta_{m}-\gamma_{1, \alpha}} & \cdots & -\frac{1}{\eta_{m}-\gamma_{\hat{n}, \alpha}} \\
\frac{1}{\theta_{1}+\beta_{1, \alpha}} & \cdots & \frac{1}{\theta_{1}+\beta_{\hat{m}, \alpha}} & -\frac{1}{\theta_{1}+\gamma_{1, \alpha}} & \cdots & -\frac{1}{\theta_{1}+\gamma_{\hat{n}, \alpha}} \\
\vdots & \ddots & \vdots & \vdots & \ddots & \vdots \\
\frac{1}{\theta_{n}+\beta_{1, \alpha}} & \cdots & \frac{1}{\theta_{n}+\beta_{\hat{m}, \alpha}} & -\frac{1}{\theta_{n}+\gamma_{1, \alpha}} & \cdots & -\frac{1}{\theta_{n}+\gamma_{\hat{n}, \alpha}}
\end{array}\right) .
$$

This concludes the proof.

\section{C.3 Proof of Proposition A.3: The pricing of Canadized one-touch digital puts in the D-MEM framework}

Following the same line of arguments as in Appendix C.2, we compute the price of the one-touch digital put in Equation (8) by solving the PIDE

$$
-\frac{\partial P}{\partial \tau}(x, \tau)+\frac{\sigma^{2}}{2} \frac{\partial^{2} P}{\partial x^{2}}(x, \tau)+\bar{\mu} \frac{\partial P}{\partial x}(x, \tau)+\int_{-\infty}^{+\infty}[P(x+y, \tau)-P(x, \tau)] \nu(y) d y=0 .
$$

The boundary and initial conditions are: $\lim _{x \uparrow+\infty} P(x, \tau)=0, \lim _{x \downarrow \kappa} P(x, \tau)=1$, and $\lim _{\tau \downarrow 0} P(x, \tau)=$ $\mathbb{1}_{\{x<\kappa\}}$. Unless otherwise stated, we keep the same notation as in Appendix C.2. The proof is similar to the one provided for Canadized European digital puts. First, we introduce the displaced variable $y \mapsto y^{\prime}:=y-\xi$. In a second step, we apply the LCT. A notable difference is that, due to a path-dependent payoff, we have two different regions in the case of one-touch digital puts: the continuation region $(x>\kappa)$ and the stopping region $(x \leq \kappa)$.

The LCT of the PIDE C.30 yields the following OIDE in the continuation region

$$
\frac{\sigma^{2}}{2} \frac{d^{2} \tilde{P}}{d x^{2}}(x, \alpha)+\bar{\mu} \frac{d \tilde{P}}{d x}(x, \alpha)-(\lambda+\alpha) \tilde{P}(x, \alpha)+\lambda \int_{-\infty}^{+\infty} \tilde{P}\left(x+\xi+y^{\prime}, \alpha\right) f_{Y}\left(y^{\prime}\right) d y^{\prime}=0 .
$$

The initial condition is again absorbed into the resulting OIDE. The boundary conditions are given 
by

$$
\begin{aligned}
& \lim _{x \downarrow \kappa} \tilde{P}(x, \alpha)=1, \\
& \lim _{x \uparrow+\infty} \tilde{P}(x, \alpha)=0 .
\end{aligned}
$$

We follow the same steps as in the case of digital puts and introduce the ansatz

$$
\tilde{P}(x, \alpha)=\mathbb{1}_{\{x>\kappa\}} \sum_{l=1}^{\hat{n}} v_{l}^{\prime} e^{\gamma_{l, \alpha}(x-\kappa-\xi)}+\mathbb{1}_{\{x \leq \kappa\}},
$$

where $v_{l}^{\prime}:=v_{l} e^{\gamma_{l, \alpha} \xi}$, for $l=1, \cdots, \hat{n}$. The two derivative terms in Equation (C.31) are

$$
\begin{aligned}
& \frac{d \tilde{P}}{d x}(x, \alpha)=\sum_{l=1}^{\hat{n}} v_{l}^{\prime} \gamma_{l, \alpha} e^{\gamma_{l, \alpha}(x-\kappa-\xi)} \\
& \frac{d^{2} \tilde{P}}{d x^{2}}(x, \alpha)=\sum_{l=1}^{\hat{n}} v_{l}^{\prime} \gamma_{l, \alpha}^{2} e^{\gamma_{l, \alpha}(x-\kappa-\xi)}
\end{aligned}
$$

The integral term in Equation C.31 becomes

$$
\begin{aligned}
\mathscr{J}: & =\int_{-\infty}^{+\infty} \tilde{P}\left(x+\xi+y^{\prime}, \alpha\right) f_{Y}\left(y^{\prime}\right) d y^{\prime} \\
& =\frac{\lambda_{+}}{\lambda} \sum_{l=1}^{\hat{n}} \sum_{i=1}^{m} \int_{0}^{+\infty} p_{i} \eta_{i} v_{l}^{\prime} e^{\gamma_{l, \alpha}(x-\kappa)} e^{\left(\gamma_{l, \alpha}-\eta_{i}\right) y^{\prime}} d y^{\prime} \\
& +\frac{\lambda_{-}}{\lambda} \sum_{j=1}^{n} \int_{-\infty}^{\kappa-x} q_{j} \theta_{j} e^{\theta_{j} y^{\prime}} d y^{\prime} \\
& +\frac{\lambda_{-}}{\lambda} \sum_{l=1}^{\hat{n}} \sum_{j=1}^{n} \int_{\kappa-x}^{0} q_{j} \theta_{j} v_{l}^{\prime} e^{\gamma_{l, \alpha}(x-\kappa)} e^{\left(\gamma_{l, \alpha}+\theta_{j}\right) y^{\prime}} d y^{\prime} .
\end{aligned}
$$

Evaluating the integrals gives us the condition

$$
\sum_{l=1}^{\hat{n}} v_{l} e^{\gamma_{l, \alpha}(x-\kappa)}\left(\Psi^{\prime}\left(\gamma_{l, \alpha}\right)-\alpha\right)-\sum_{l=1}^{n} \lambda_{-} q_{l} \theta_{l} e^{\theta_{l}(\kappa-x)}\left(\sum_{j=1}^{\hat{n}} \frac{v_{j}}{\theta_{l}+\gamma_{j, \alpha}}-\frac{1}{\theta_{l}}\right)=0 .
$$

Following the same logic as in the previous proof, the following $n$ linear equations emerge:

$$
\sum_{j=1}^{\hat{n}} \frac{v_{j}}{\theta_{l}+\gamma_{j, \alpha}}=\frac{1}{\theta_{l}}, \text { for } l=1, \ldots, n .
$$

We close the system with the value matching condition at the boundary between the stopping and the continuation region, which is

$$
\sum_{j=1}^{\hat{n}} v_{j}=1
$$

Finally, we collect the conditions (C.37) and C.38, and obtain the matrix equation

$$
\mathbf{B v}=\mathbf{b}
$$

where

$$
\mathbf{v}=\left(v_{1}, v_{2}, \ldots, v_{\hat{n}}\right)^{\prime}
$$


is an $\hat{n}$-dimensional column vector, and

$$
\mathbf{b}=\left(1, \frac{1}{\theta_{1}}, \ldots, \frac{1}{\theta_{n}}\right)^{\prime}
$$

is an $(n+1)$-dimensional column vector. Finally, the matrix $\mathbf{B}$ is the $(n+1) \times \hat{n}$-dimensional matrix given by

$$
\mathbf{B}=\left(\begin{array}{ccc}
1 & \cdots & 1 \\
\frac{1}{\theta_{1}+\gamma_{1, \alpha}} & \cdots & \frac{1}{\theta_{1}+\gamma_{\hat{n}, \alpha}} \\
\vdots & \ddots & \vdots \\
\frac{1}{\theta_{n}+\gamma_{1, \alpha}} & \cdots & \frac{1}{\theta_{n}+\gamma_{\hat{n}, \alpha}}
\end{array}\right)
$$

This completes the proof.

\section{C.4 Proof of Proposition A.4: First-passage disentanglement of Canadized one- touch digital puts in the D-MEM framework}

We showed in Proposition A.3 that a Canadized one-touch digital put satisfies the OIDE (C.31) with the boundary conditions C.32. It follows from the Feynman-Kac formula that we can express the price of a Canadized one-touch digital put as

$$
\tilde{P}(x, \alpha)=\mathbb{E}_{t}\left[e^{-\alpha\left(\tau_{\kappa}-t\right)}\right]
$$

where $\tau_{\kappa}$ is the first-passage time (from above) of the barrier level $\kappa$ for the log-price process $X_{t}$ :

$$
\tau_{\kappa}:=\inf \left\{u \geq t: X_{u} \leq \kappa\right\}
$$

The mathematical formalism of the relation between the OIDE system C.31-C.32 and the expectation in Equation C.43 can be derived by closely following the proof of theorem 3.1 in Kou and Wang (2003), pp. 509-512, and theorem 3.3 in Cai and Kou (2011), p. 2072. Since we are studying the Laplace transform of the first-passage time to a lower boundary, and the aforementioned papers study the Laplace transform of the first-passage time to an upper boundary, the formal proof is omitted in our paper.

Following the notation in theorem 3 in Leippold and Vasiljević (2017), pp. 9-10, 29-31, the set $\mathscr{E}_{\mathcal{D}}:=\left\{X_{\tau_{\kappa}}=\kappa\right\}$ represents all possible events of a stopping of the process $X_{t}$ exactly at the barrier 
$\kappa$, i.e., a stopping due to the diffusion. Similarly, we denote by $\mathscr{E}_{\mathcal{J}}:=\left\{X_{\tau_{\kappa}}<\kappa\right\}$ the set of all possible events of a stopping due to overshooting of the barrier level $\kappa$ by the process $X_{t}$, i.e., a stopping due to the jumps. The price of the Canadized one-touch digital put given in Equation (C.43) can be orthogonally decomposed as

$$
\tilde{P}(x, \alpha)=\mathbb{E}_{t}\left[e^{-\alpha\left(\tau_{\kappa}-t\right)} \mathbb{1}_{\mathscr{E}_{\mathcal{D}}}\right]+\mathbb{E}_{t}\left[e^{-\alpha\left(\tau_{\kappa}-t\right)} \mathbb{1}_{\mathscr{E}_{\mathcal{J}}}\right]
$$

It will therefore suffice to compute either the diffusion or the jump contribution, since $\tilde{P}(x, \alpha)=$ $\tilde{P}_{D}(x, \alpha)+\tilde{P}_{J}(x, \alpha)$, and the (total) price of a one-touch digital put is given in Proposition A.3.

Following the solution procedure in Appendix C.2 and Appendix C.3, we introduce the displaced variables $x \mapsto x=x+\xi$ and $y \mapsto y^{\prime}:=y-\xi$, and define $\kappa:=\kappa+\xi$. Subsequently, we apply the LCT. Therefore, to compute, e.g., the diffusion contribution $\tilde{P}_{D}(x, \alpha)$, we have to solve the OIDE

$$
\frac{\sigma^{2}}{2} \frac{d^{2} \tilde{P}_{D}}{d x^{2}}(x, \alpha)+\bar{\mu} \frac{d \tilde{P}_{D}}{d x}(x, \alpha)-(\lambda+\alpha) \tilde{P}_{D}(x, \alpha)+\lambda \int_{-\infty}^{+\infty} \tilde{P}_{D}\left(x+y^{\prime}, \alpha\right) f_{Y}\left(y^{\prime}\right) d y^{\prime}=0,
$$

which is the same as Equation C.31. The boundary conditions C.32 remain unchanged as well. However, the diffusion contribution in the interior of the stopping region, i.e., for $x<\kappa$, is zero. This is a consequence of the fact that a stopping due to the diffusion can happen only at the boundary, i.e., almost surely a diffusion will not generate an overshoot. Therefore, we use the following ansatz:

$$
\tilde{P}_{D}(x, \alpha)=\mathbb{1}_{\{x>\kappa\}} \sum_{j=1}^{\hat{n}} \delta_{j} e^{\gamma_{j, \alpha}(x-\kappa)}+\mathbb{1}_{\{x=\kappa\}} .
$$

After some algebra, we obtain the conditions that the summation coefficients $\left\{\delta_{j}\right\}_{j=1, \ldots, \hat{n}}$ have to satisfy:

$$
\left\{\begin{array}{l}
\sum_{j=1}^{\hat{n}} \frac{\delta_{j}}{\theta_{l}+\gamma_{j, \alpha}}=0, \text { for } l=1, \ldots, n, \\
\sum_{j=1}^{\hat{n}} \delta_{j}=1
\end{array}\right.
$$

We rewrite these conditions in the matrix form

$$
\mathbf{M}_{D} \boldsymbol{\delta}=\boldsymbol{\epsilon}_{D}, \quad \boldsymbol{\delta}=\left(\delta_{1}, \delta_{2}, \ldots, \delta_{\hat{n}}\right)^{\prime}
$$

and

$$
\boldsymbol{\epsilon}_{D}=(1, \underbrace{0, \ldots, 0}_{n \text { times }})^{\prime}
$$


is an $(n+1)$-dimensional column vector. The matrix $\mathbf{M}_{D}$ is the $(n+1) \times \hat{n}$-dimensional matrix which is the same as the matrix $\mathbf{B}$, which is given in Equation C.42. By analogy, it can be shown the summation coefficients $\left\{\iota_{i}\right\}_{j=1, \ldots, \hat{m}}$ can be computed as the solution of the matrix equation

$$
\mathbf{M}_{J} \iota=\boldsymbol{\epsilon}_{J}, \quad \iota=\left(\iota_{1}, \iota_{2}, \ldots, \iota_{\hat{m}}\right)^{\prime}
$$

and

$$
\boldsymbol{\epsilon}_{J}=\left(0, \frac{1}{\theta_{1}}, \ldots, \frac{1}{\theta_{n}}\right)^{\prime}
$$

is an $(n+1)$-dimensional column vector. The matrix $\mathbf{M}_{J}$ is the $(n+1) \times \hat{n}$-dimensional matrix which is identical to the matrix $\mathbf{M}_{D}$. This concludes the proof. 
Tables 
Table 1: S\&P 100 index options data, March 2001-August 2014. Descriptive statistics for near-themoney (NTM) and out-of-the-money (OTM) S\&P 100 American put options with maturities of up to ten days. The data was obtained from OptionMetrics and filtered according to standard criteria. The dataset comprises closing quotes of liquid put options sampled monthly. There are 162 observation dates in total. We report the number of option contracts traded (Panel A), the average quoted price (Panel B) and the average implied volatility (Panel C). Each statistic is computed for three different maturity bins and four different moneyness bins, as well as for the entire sample, i.e., aggregated across the maturity and the moneyness dimension. DTM stands for days to maturity.

\begin{tabular}{|c|c|c|c|c|}
\hline \multicolumn{5}{|c|}{ Panel A: Number of contracts across moneyness and maturity } \\
\hline Moneyness & $\mathrm{DTM} \leq 5$ & $5<\mathrm{DTM} \leq 9$ & $\mathrm{DTM}=10$ & All \\
\hline$K / F<0.94$ & 78 & 81 & 1,207 & 1,366 \\
\hline $0.94<K / F<0.97$ & 173 & 22 & 437 & 632 \\
\hline $0.97<K / F<1.00$ & 311 & 26 & 450 & 787 \\
\hline $1.00<K / F<1.03$ & $\underline{164}$ & $\underline{27}$ & $\underline{435}$ & $\underline{626}$ \\
\hline All & 726 & 156 & 2,529 & 3,411 \\
\hline \multicolumn{5}{|c|}{ Panel B: Average quoted price across moneyness and maturity } \\
\hline Moneyness & $\mathrm{DTM} \leq 5$ & $5<\mathrm{DTM} \leq 9$ & $\mathrm{DTM}=10$ & All \\
\hline $\mathrm{K} / \mathrm{F}<0.94$ & 0.39 & 0.42 & 0.65 & 0.62 \\
\hline $0.94<\mathrm{K} / \mathrm{F}<0.97$ & 0.70 & 1.51 & 1.97 & 1.60 \\
\hline $0.97<\mathrm{K} / \mathrm{F}<1.00$ & 2.16 & 3.54 & 4.90 & 3.77 \\
\hline $1.00<\mathrm{K} / \mathrm{F}<1.03$ & $\underline{8.78}$ & $\underline{11.84}$ & $\underline{13.02}$ & $\underline{11.86}$ \\
\hline All & $\overline{3.11}$ & $\overline{3.07}$ & $\overline{3.76}$ & $\overline{3.59}$ \\
\hline \multicolumn{5}{|c|}{ Panel C: Average implied volatility across moneyness and maturity } \\
\hline Moneyness & $\mathrm{DTM} \leq 5$ & $5<\mathrm{DTM} \leq 9$ & $\mathrm{DTM}=10$ & All \\
\hline$K / F<0.94$ & 0.4985 & 0.3721 & 0.3930 & 0.3978 \\
\hline $0.94<K / F<0.97$ & 0.3444 & 0.2467 & 0.2452 & 0.2724 \\
\hline $0.97<K / F<1.00$ & 0.2343 & 0.1960 & 0.2088 & 0.2184 \\
\hline $1.00<K / F<1.03$ & $\underline{0.2475}$ & $\underline{0.1988}$ & $\underline{0.1987}$ & $\underline{0.2115}$ \\
\hline All & $\overline{0.2920}$ & $\overline{0.2951}$ & $\overline{0.3014}$ & $\overline{0.2991}$ \\
\hline
\end{tabular}




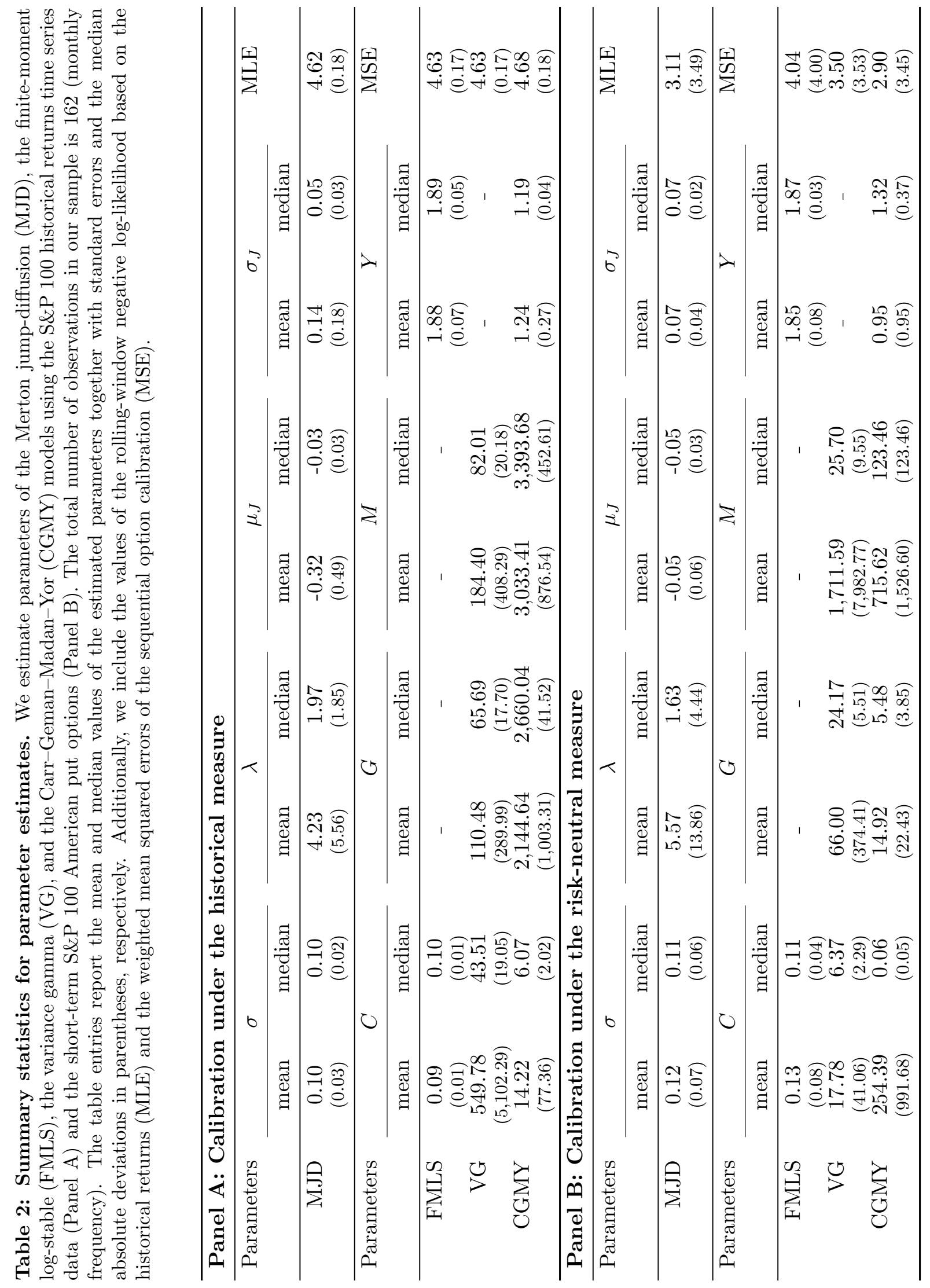


Table 3: Model performance (pairwise comparison). The table entries report the values of Vuong (1989)'s closeness test statistic for pairwise equivalence of non-nested models in terms of model performance. The statistics are computed for the pairwise combinations of the Merton jump-diffusion (MJD), the finitemoment log-stable (FMLS), the variance gamma (VG), and the Carr-Geman-Madan-Yor (CGMY) models. Panel A (Panel B) reports the pairwise statistics for the maximum likelihood estimation (weighted non-linear least squares estimation) under the historical (risk-neutral) measure. The null hypothesis is that there is no difference between the two models in terms of the closeness to the true data generating process, and the alternative is that one model is closer. Positive values of test statistic larger than 1.65 (2.32) imply, for the given pair of models, the domination of the first model over the second one at the confidence level of $95 \%$ (99\%). Negative values of the test statistic less than $-1.65(-2.32)$ imply domination of the second model over the first model at the confidence level of $95 \%(99 \%)$.

\begin{tabular}{|c|c|c|c|c|}
\hline \multicolumn{5}{|c|}{ Panel A: Pairwise model comparison under the historical measure } \\
\hline Model & MJD & FMLS & VG & CGMY \\
\hline MJD & - & -1.80 & -2.91 & 11.87 \\
\hline FMLS & - & - & -1.65 & 17.77 \\
\hline VG & - & - & - & 16.68 \\
\hline \multicolumn{5}{|c|}{ Panel B: Pairwise model comparison under the risk-neutral measure } \\
\hline Model & MJD & FMLS & VG & CGMY \\
\hline MJD & - & 7.19 & 6.05 & -5.26 \\
\hline FMLS & - & - & -3.61 & -8.80 \\
\hline VG & - & - & - & -8.60 \\
\hline
\end{tabular}


Table 4: VaR and iVaR multiples. The table reports average, median, and maximum multiples of the endof-horizon value at risk (VaR) and the intra-horizon value at risk (iVaR), computed for $\chi=99.0 \%$ and $99.9 \%$ confidence levels with a 10-day monitoring horizon, for the Merton jump-diffusion (MJD), the finite-moment log-stable (FMLS), the variance gamma (VG), and the Carr-Geman-Madan-Yor (CGMY) models under the historical and option-implied measures. The analysis is based on the time series of S\&P 100 historical returns and the short-term S\&P 100 American put options over the period March 2001-August 2014. The models are re-estimated on a monthly basis, hence generating the total number of 162 observation dates in the sample. For each considered model, the multiples are computed as the ratios of the respective VaR and iVaR estimates and the benchmark VaR value, which is given as either the $1 \%$ or $0.1 \%$ quantile of the normal distribution $\mathcal{N}\left(0, \hat{\sigma}^{2}\right)$, where $\hat{\sigma}$ is the standard deviation of the (de-meaned) time series of S\&P 100 historical returns. We also report (in parentheses) the VaR and the iVaR multiples of our jump models with respect to the VaR estimates based on the filtered historical simulation (FHS) approach.

\begin{tabular}{|c|c|c|c|c|}
\hline \multicolumn{5}{|c|}{ Panel A: Historical VaR \& iVaR multiples } \\
\hline$\chi=\mathbf{9 9 . 0} \%$ & MJD & FMLS & VG & CGMY \\
\hline Avg. VaR & $1.11(0.73)$ & $1.20(0.79)$ & $1.20(0.79)$ & $1.00(0.66)$ \\
\hline Med. VaR & $1.11(0.73)$ & $1.19(0.76)$ & $1.21(0.77)$ & $0.99(0.65)$ \\
\hline Max. VaR & $1.99(1.24)$ & $1.70(1.13)$ & $1.85(1.15)$ & $1.26(0.87)$ \\
\hline Avg. iVaR & $1.19(0.79)$ & $1.29(0.85)$ & $1.29(0.85)$ & $1.07(0.71)$ \\
\hline Med. iVaR & $1.21(0.78)$ & $1.28(0.82)$ & $1.30(0.83)$ & $1.06(0.70)$ \\
\hline Max. iVaR & $2.16(1.35)$ & $1.83(1.22)$ & $1.99(1.24)$ & $1.35(0.93)$ \\
\hline$\chi=99.9 \%$ & MJD & FMLS & VG & CGMY \\
\hline Avg. VaR & $1.38(0.85)$ & $1.99(1.23)$ & $1.32(0.81)$ & $1.00(0.62)$ \\
\hline Med. VaR & $1.39(0.81)$ & $2.03(1.20)$ & $1.34(0.80)$ & $0.98(0.61)$ \\
\hline Max. VaR & $2.47(1.63)$ & $3.43(2.11)$ & $2.09(1.20)$ & $2.65(1.02)$ \\
\hline Avg. iVaR & $1.43(0.88)$ & $2.07(1.28)$ & $1.37(0.85)$ & $1.04(0.65)$ \\
\hline Med. iVaR & $1.43(0.84)$ & $2.11(1.25)$ & $1.39(0.83)$ & $1.02(0.64)$ \\
\hline Max. iVaR & $2.59(1.71)$ & $3.52(2.17)$ & $2.19(1.25)$ & $1.73(1.08)$ \\
\hline \multicolumn{5}{|c|}{ Panel B: Option-implied VaR \& iVaR multiples } \\
\hline$\chi=99.0 \%$ & MJD & FMLS & VG & CGMY \\
\hline Avg. VaR & $1.61(1.06)$ & $1.69(1.11)$ & $1.87(1.23)$ & $1.81(1.19)$ \\
\hline Med. VaR & $1.37(1.94)$ & $1.35(0.89)$ & $1.51(1.01)$ & $1.49(0.99)$ \\
\hline Max. VaR & $6.57(3.93)$ & $7.52(4.50)$ & $7.41(4.43)$ & $7.61(4.55)$ \\
\hline Avg. iVaR & $1.69(1.11)$ & $1.83(1.20)$ & $2.02(1.32)$ & $1.95(1.28)$ \\
\hline Med. iVaR & $1.42(0.96)$ & $1.46(0.96)$ & $1.65(1.08)$ & $1.61(1.07)$ \\
\hline Max. iVaR & $6.82(4.07)$ & $8.04(4.81)$ & $7.90(4.72)$ & $8.12(4.86)$ \\
\hline$\chi=99.9 \%$ & MJD & FMLS & VG & CGMY \\
\hline Avg. VaR & $2.28(1.40)$ & $2.87(1.77)$ & $2.15(1.31)$ & $2.47(1.51)$ \\
\hline Med. VaR & $2.15(1.33)$ & $2.58(1.57)$ & $1.82(1.15)$ & $2.27(1.38)$ \\
\hline Max. VaR & $7.15(3.28)$ & $8.11(4.19)$ & $7.36(3.38)$ & $9.96(5.63)$ \\
\hline Avg. iVaR & $2.35(1.44)$ & $2.99(1.84)$ & $2.25(1.38)$ & $2.57(1.57)$ \\
\hline Med. iVaR & $2.22(1.35)$ & $2.69(1.65)$ & $1.92(1.21)$ & $2.36(1.43)$ \\
\hline Max. iVaR & $7.29(3.35)$ & $8.48(4.20)$ & $7.62(3.50)$ & $9.96(5.63)$ \\
\hline
\end{tabular}


Table 5: VaR and iVaR backtesting. The table summarizes the backtesting results for the end-of-horizon value at risk (VaR) and the intra-horizon value at risk (iVaR), computed at the confidence level of $\chi=99.0 \%$, and for the regulatory 10-day monitoring period, for the Merton jump-diffusion (MJD), the finite-moment log-stable (FMLS), the variance gamma (VG), and the Carr-Geman-Madan-Yor (CGMY) models under the historical and the option-implied measures. Additionally, we provide test statistics for the filtered historical simulation (FHS). The table reports the likelihood ratio (LR) and the $p$-value ( $p$-val.) for the following backtesting procedures: the Time Until First Failure test (TUFF), the Unconditional Coverage test (UC), the Independence Coverage test (IC), and the Conditional Coverage test (CC).

\begin{tabular}{|c|c|c|c|c|c|c|c|c|c|}
\hline \multicolumn{10}{|c|}{ Panel A: Historical VaR backtesting } \\
\hline \multirow[t]{2}{*}{ Method } & \multirow[t]{2}{*}{$\#$} & \multicolumn{2}{|c|}{ TUFF } & \multicolumn{2}{|c|}{$\mathrm{UC}$} & \multicolumn{2}{|c|}{$\mathrm{IC}$} & \multicolumn{2}{|c|}{$\mathrm{CC}$} \\
\hline & & LR & $p$-val. & LR & $p$-val. & LR & $p$-val. & LR & $p$-val. \\
\hline FHS & 6 & 1.9225 & 0.1652 & 6.9629 & 0.0083 & 6.5053 & 0.0108 & 13.4682 & 0.0012 \\
\hline MJD & 7 & 9.2103 & 0.0024 & 9.7092 & 0.0018 & 5.8424 & 0.0156 & 15.5525 & 0.0004 \\
\hline FMLS & 6 & 1.9225 & 0.1652 & 6.9629 & 0.0083 & 6.5053 & 0.0108 & 13.4682 & 0.0012 \\
\hline VG & 7 & 9.2103 & 0.0024 & 9.7762 & 0.0018 & 5.8195 & 0.0158 & 15.5957 & 0.0004 \\
\hline CGMY & 7 & 9.2103 & 0.0024 & 9.7762 & 0.0018 & 5.8195 & 0.0158 & 15.5957 & 0.0004 \\
\hline \multicolumn{10}{|c|}{ Panel B: Option-implied VaR backtesting } \\
\hline \multirow[t]{2}{*}{ Method } & $\#$ & \multicolumn{2}{|c|}{ TUFF } & \multicolumn{2}{|c|}{$\mathrm{UC}$} & \multicolumn{2}{|c|}{$\mathrm{IC}$} & \multicolumn{2}{|c|}{$\mathrm{CC}$} \\
\hline & & LR & $p$-val. & LR & $p$-val. & LR & $p$-val. & LR & $p$-val. \\
\hline MJD & 1 & 1.9225 & 0.1652 & 0.3010 & 0.5832 & 0.0123 & 0.9951 & 0.3133 & 0.8550 \\
\hline FMLS & 0 & 0.2931 & 0.5882 & 3.2965 & 0.0694 & 0.0000 & 1.0000 & 3.2965 & 0.1925 \\
\hline VG & 0 & 0.2931 & 0.5882 & 3.2965 & 0.0694 & 0.0000 & 1.0000 & 3.2965 & 0.1925 \\
\hline CGMY & 1 & 0.1863 & 0.6660 & 0.2931 & 0.5882 & 0.0123 & 0.9619 & 0.3055 & 0.8584 \\
\hline \multicolumn{10}{|c|}{ Panel C: Historical iVaR backtesting } \\
\hline \multirow[t]{2}{*}{ Method } & $\#$ & \multicolumn{2}{|c|}{ TUFF } & \multicolumn{2}{|c|}{$\mathrm{UC}$} & \multicolumn{2}{|c|}{$\mathrm{IC}$} & \multicolumn{2}{|c|}{$\mathrm{CC}$} \\
\hline & & LR & $p$-val. & LR & $p$-val. & LR & $p$-val. & $\mathrm{LR}$ & $p$-val. \\
\hline FHS & 8 & 3.5893 & 0.0582 & 12.8887 & 0.0003 & 9.0679 & 0.0026 & 21.9566 & $<10^{-4}$ \\
\hline MJD & 11 & 9.2103 & 0.0024 & 23.5822 & $<10^{-4}$ & 10.2772 & 0.0013 & 33.8594 & $<10^{-4}$ \\
\hline FMLS & 8 & 3.5893 & 0.0582 & 12.8887 & 0.0003 & 9.0679 & 0.0026 & 21.9566 & $<10^{-4}$ \\
\hline VG & 9 & 3.5893 & 0.0582 & 16.2644 & 0.0001 & 7.4684 & 0.0063 & 23.7338 & $<10^{-4}$ \\
\hline CGMY & 9 & 3.5893 & 0.0582 & 16.2644 & 0.0001 & 7.4684 & 0.0063 & 23.7338 & $<10^{-4}$ \\
\hline \multicolumn{10}{|c|}{ Panel D: Option-implied iVaR backtesting } \\
\hline \multirow[t]{2}{*}{ Method } & $\#$ & \multicolumn{2}{|c|}{ TUFF } & \multicolumn{2}{|c|}{$\mathrm{UC}$} & \multicolumn{2}{|c|}{$\mathrm{IC}$} & \multicolumn{2}{|c|}{$\mathrm{CC}$} \\
\hline & & LR & $p$-val. & LR & $p$-val. & LR & $p$-val. & $\mathrm{LR}$ & $p$-val. \\
\hline MJD & 1 & 0.1426 & 0.7057 & 0.3010 & 0.5832 & 0.0123 & 0.9118 & 0.3133 & 0.8550 \\
\hline FMLS & 0 & 0.2931 & 0.5882 & 3.2965 & 0.0694 & 0.0000 & 1.0000 & 3.2965 & 0.1925 \\
\hline VG & 0 & 0.2931 & 0.5882 & 3.2965 & 0.0694 & 0.0000 & 1.0000 & 3.2965 & 0.1925 \\
\hline CGMY & 1 & 0.1863 & 0.6660 & 0.2931 & 0.5882 & 0.0123 & 0.9115 & 0.3055 & 0.8584 \\
\hline
\end{tabular}


Figures 
Figure 1: First-passage disentanglement of the iVaR (10-day horizon, 99.0\% confidence level). For each considered model, the Merton jump-diffusion (MJD), the finite-moment log-stable (FMLS), the variance gamma (VG), and the Carr-Geman-Madan-Yor (CGMY) models, which are given in Panels A, $\mathrm{B}, \mathrm{C}$, and D, respectively, we plot the time evolution of the jump contribution to the 10-day intra-horizon value at risk (iVaR) calculated at the confidence level of 99.0\%. Our results are based on the first-passage disentanglement (FPD) approach introduced in Section C.4.
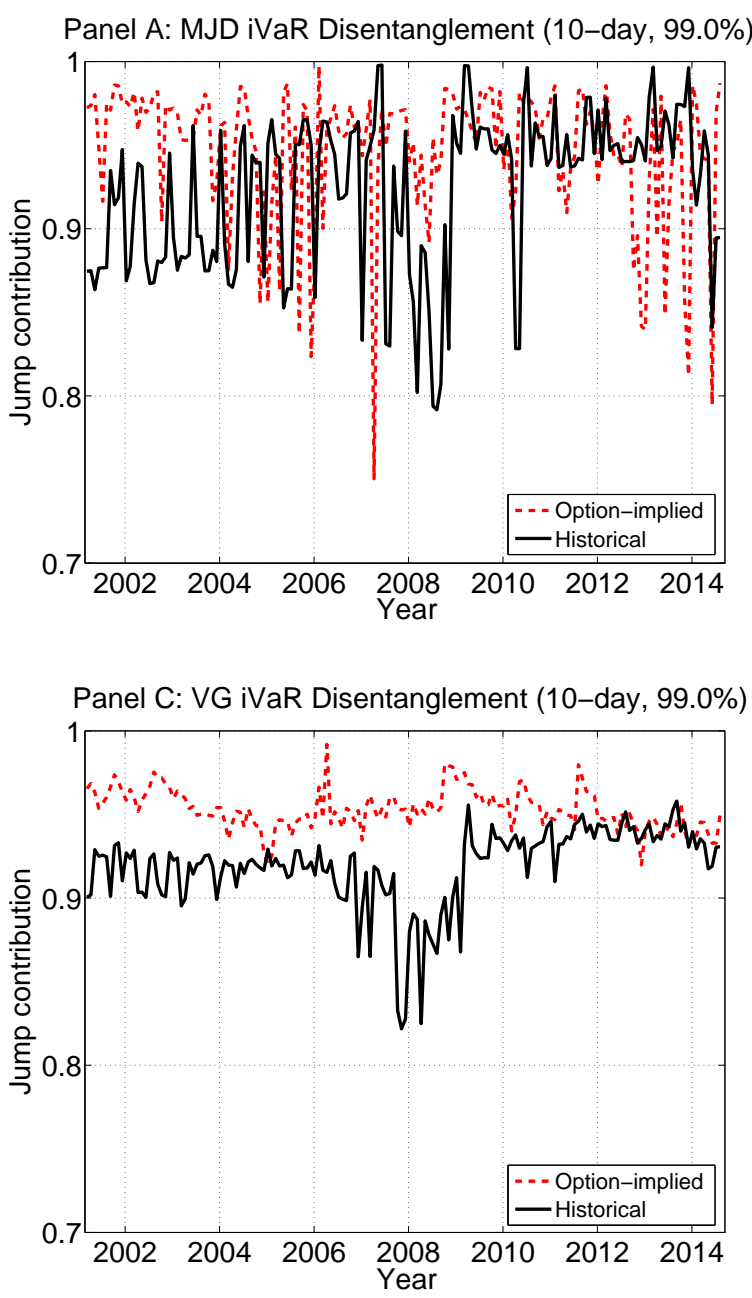
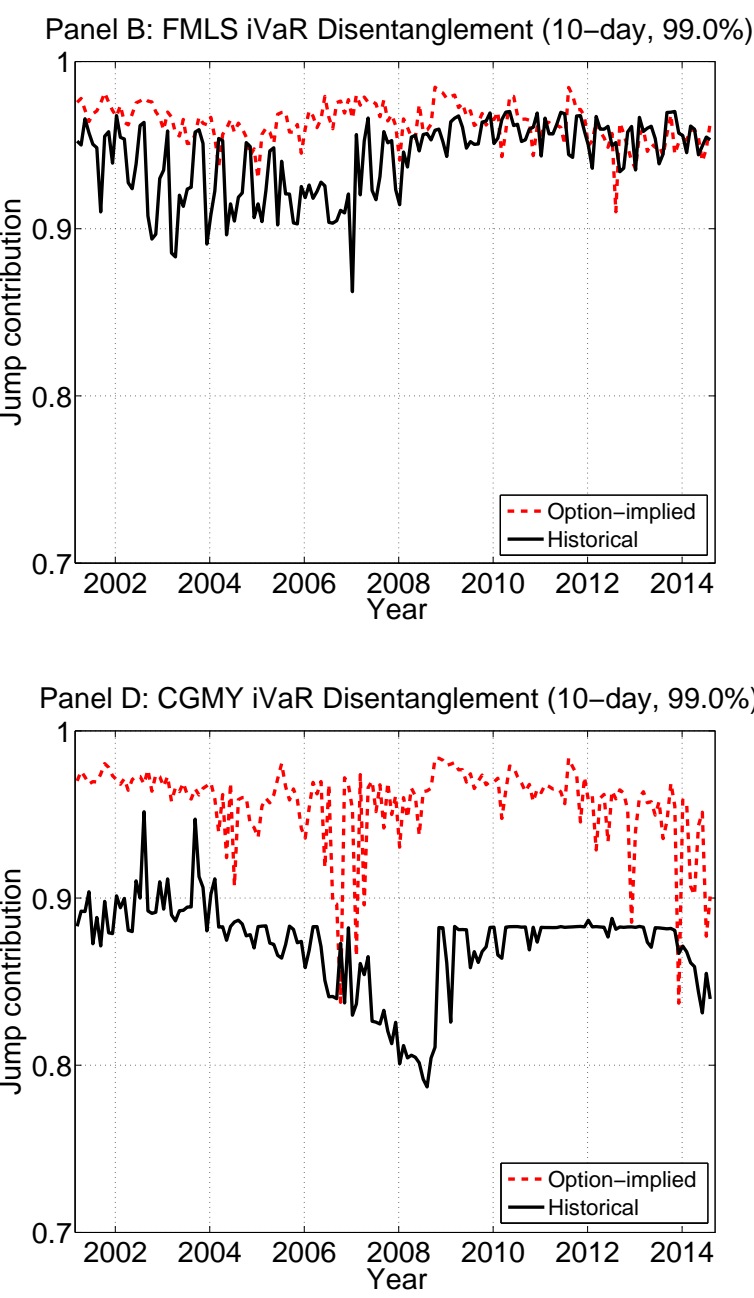
Figure 2: The impact of the probability measure on iVaR (10-day horizon, $99.0 \%$ confidence level). For each considered model, the Merton jump-diffusion (MJD), the finite-moment log-stable (FMLS), the variance gamma (VG), and the Carr-Geman-Madan-Yor (CGMY) models, we plot the time evolution of the ratio of the option-implied to historical intra-horizon values at risk (iVaR) calculated at the confidence level of $99.0 \%$, and with monitoring horizon of ten days.

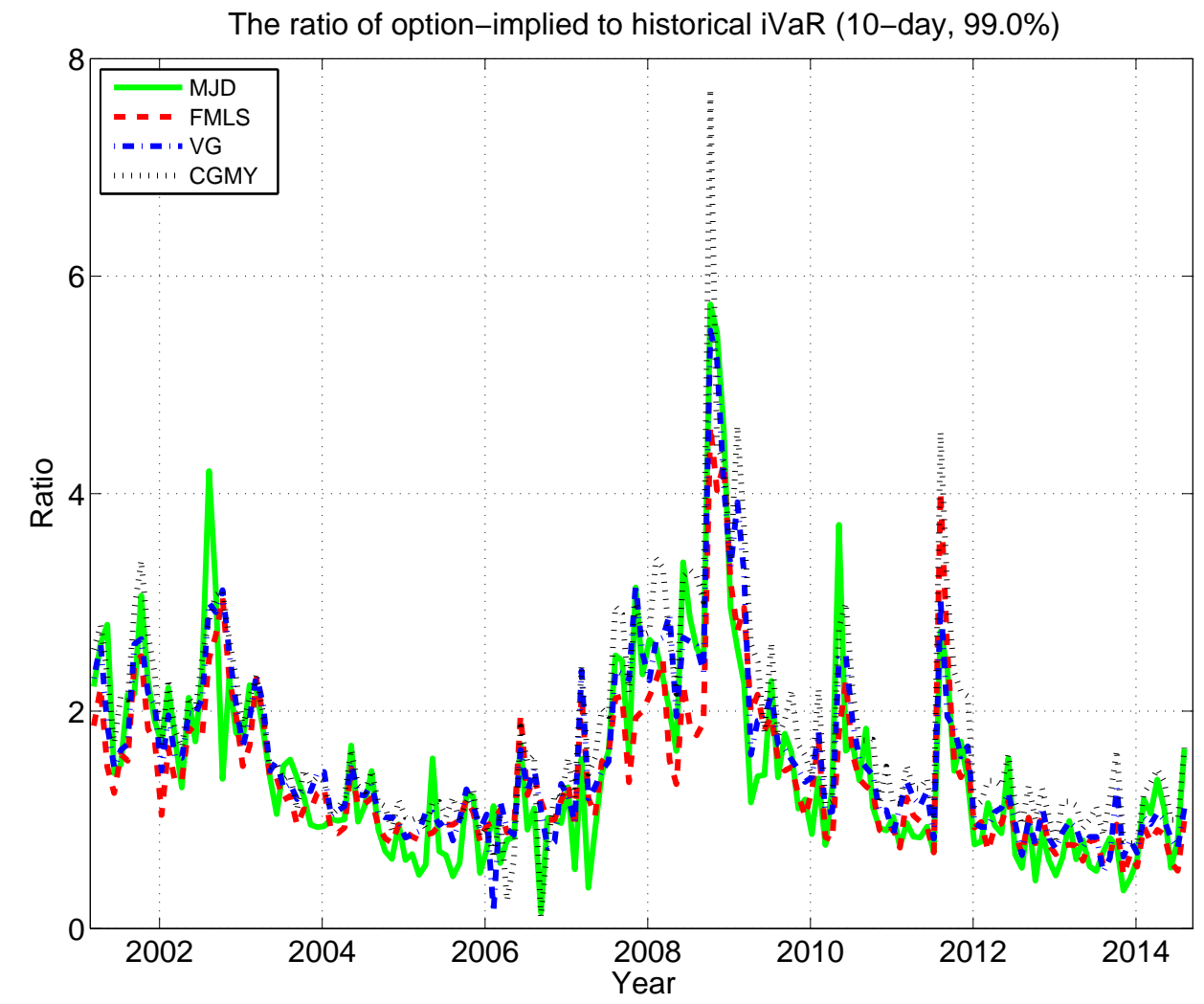


Figure 3: VaR backtesting (10-day horizon, 99.0\% confidence level). For each considered model, the Merton jump-diffusion (MJD), the finite-moment log-stable (FMLS), the variance gamma (VG), and the CarrGeman-Madan-Yor (CGMY) models, which are given in Panels A, B, C, and D, respectively, we graphically represent the time evolution of the 10-day end-of-horizon value at risk (VaR) calculated at the confidence level of $99.0 \%$ and the realized 10-day returns. We plot the estimates under both the historical and the optionimplied measures. Additionally, we include the VaR estimates based on a filtered historical simulation (FHS). The plots provide information about the ability of each model to forecast the VaR of the S\&P 100 index at the end of the regulatory 10-day horizon.
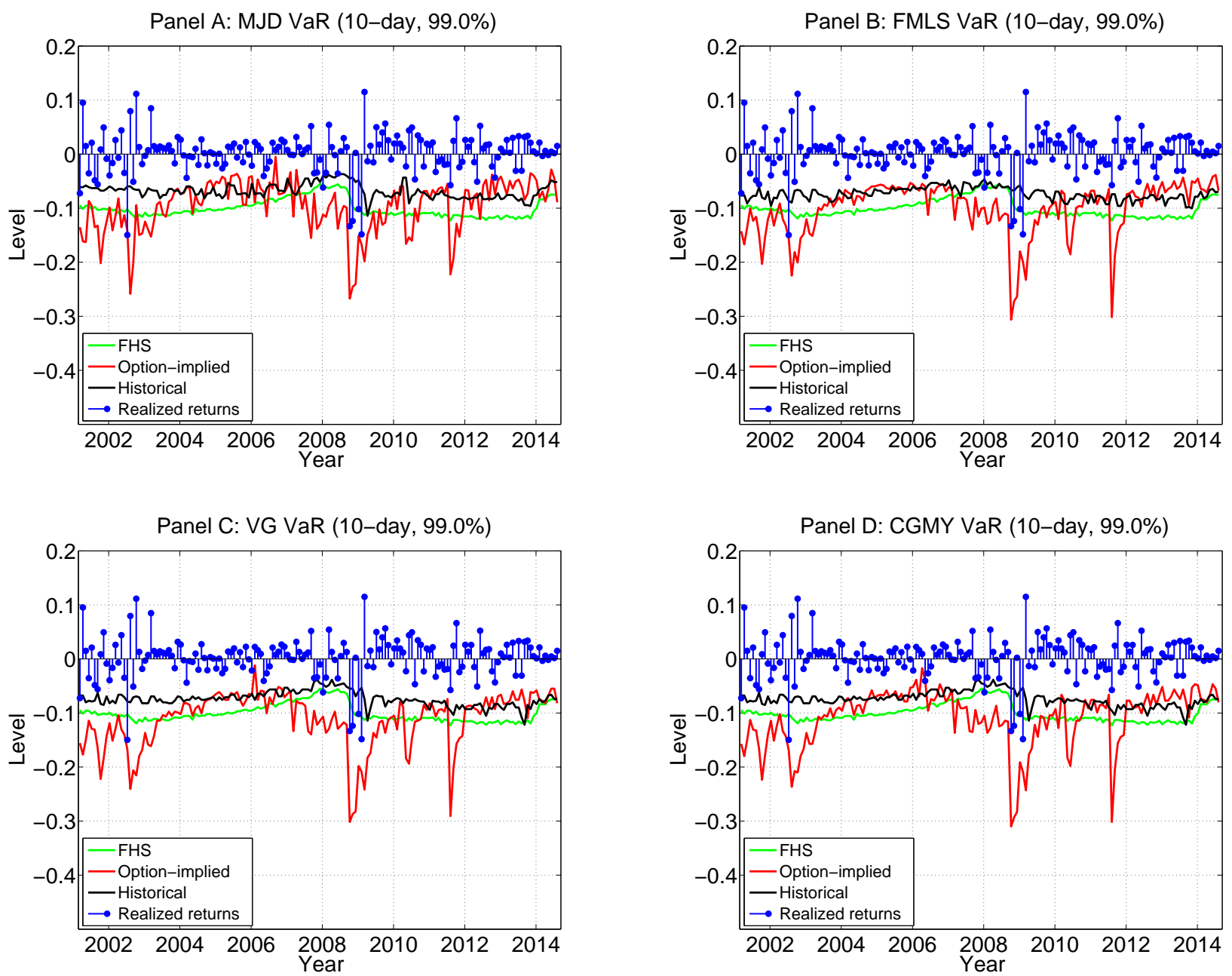
Figure 4: iVaR backtesting (10-day horizon, 99.0\% confidence level). For each considered model, the Merton jump-diffusion (MJD), the finite-moment log-stable (FMLS), the variance gamma (VG), and the Carr-Geman-Madan-Yor (CGMY) models, which are given in Panels A, B, C, and D, respectively, we graphically represent the time evolution of the 10-day intra-horizon value at risk (iVaR) calculated at the confidence level of $99.0 \%$ and the realized minimal cumulative interim 10-day returns. We plot the estimates under the historical and the option-implied measures. Additionally, we include the iVaR estimates based on a filtered historical simulation (FHS). The plots provide information about the ability of each model to forecast the iVaR of the S\&P 100 index within the regulatory 10-day horizon.
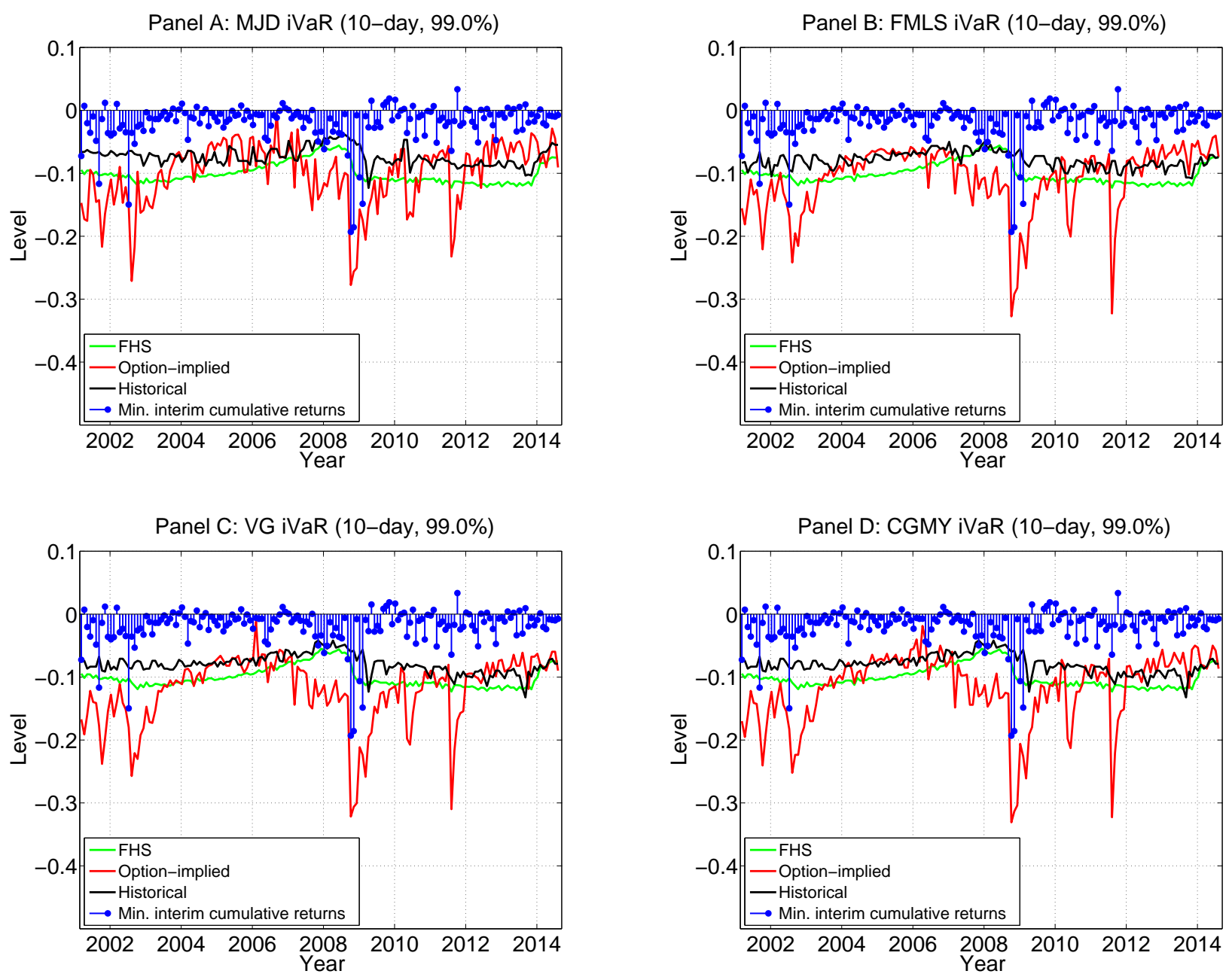